\title{
Hermite Snakes With Control of Tangents
}

\author{
Virginie Uhlmann, Student Member, IEEE, Julien Fageot, and Michael Unser, Fellow, IEEE
}

\begin{abstract}
We introduce a new model of parametric contours defined in a continuous fashion. Our curve model relies on Hermite spline interpolation and can easily generate curves with sharp discontinuities; it also grants direct access to the tangent at each location. With these two features, the Hermite snake distinguishes itself from classical spline-snake models and allows one to address certain bioimaging problems in a more efficient way. More precisely, the Hermite snake construction allows introducing sharp corners in the snake curve and designing directional energy functionals relying on local orientation information in the input image. Using the formalism of spline theory, the model is shown to meet practical requirements such as invariance to affine transformations and good approximation properties. Finally, the dependence on initial conditions and the robustness to the noise is studied on synthetic data in order to validate our Hermite snake model, and its usefulness is illustrated on real biological images acquired using brightfield, phase-contrast, differentialinterference-contrast, and scanning-electron microscopy.
\end{abstract}

Index Terms-Active contours, segmentation, Hermite splines, Hermite interpolation, bioimage analysis.

\section{INTRODUCTION}

$\mathbf{P}$ ARAMETRIC spline-snakes, a particular variety of active contours [1], [2], are widely used for the automated analysis of biomedical images. They consist of a continuous curve described by a small sets of coefficients and associated basis functions.

Segmentation with snakes is usually performed in two steps [2]. An initial configuration of the contour is first given. The snake is then optimized from this starting condition by minimizing a cost functional. Two aspects of an active contour play a significant role: the snake model describes the curve that will evolve on the image; the energy functional, usually referred to as snake energy, dictates the evolution of the curve. While remaining mostly automated, active contours allow for extensive user interaction in term of feedback and manual correction. This aspect probably explains the success of snakebased methods for segmentation. There exists an extensive literature on parametric snakes, both regarding snake model variants [3]-[5] and snake energies [6]-[9]. The formulation of the energy term is often problem-dependent.

In this paper, we propose a novel active contour that relies on Hermite-spline interpolation [10] and we show its useful-

Manuscript received July 16, 2015; revised March 1, 2016; accepted April 4, 2016. Date of publication April 6, 2016; date of current version April 29, 2016. This work was supported by the Swiss National Science Foundation under Grant 200020-121763 and Grant 200020-144355. The associate editor coordinating the review of this manuscript and approving it for publication was Dr. Alin M. Achim.

The authors are with the Biomedical Imaging Group, École Polytechnique Fédérale de Lausanne, 1015 Lausanne, Switzerland (e-mail: virginie.uhlmann@epfl.ch; julien.fageot@epfl.ch; michael.unser@epfl.ch).

Color versions of one or more of the figures in this paper are available online at http://ieeexplore.ieee.org.

Digital Object Identifier 10.1109/TIP.2016.2551363 ness for the analysis of bioimages. We present a complete and general formulation of the Hermite snake, from which a particular case (the open curve) has been briefly introduced in [11]. Our Hermite snake is reminiscent of B-spline based models [12]-[14] but benefits from the control of tangents, which makes it well-suited to bioimaging. The essential ingredients of our construction are two complementary basis functions that grant direct control on the curve and on its tangent field. This representation offers two interesting capabilities. First, it is able to reproduce sharp corners, or tips, which are much harder to obtain using classical curves. Second, the direct control of the tangents enables the design of directional energy functionals. For these reasons, the method we propose is well-suited to a wide range of segmentation tasks. We provide mathematical grounds to motivate our curve model and perform a serie of experiments to study the validity and the robustness of the proposed approach, as well as to demonstrate its strengths.

The construction of (open and closed) snake curves allowing corners and the definition of directional energy functionals has also been studied by Kimmel et al. [15] using a geodesic active contours approach. The construction of the model, the formulation of the energy, the associated optimization procedure and, ultimately, the capabilities and strengths of the resulting algorithms are however different. Geodesic snake curves as proposed in [15] are defined implicitly. They allow for topological changes but have limited potential for manual interactions. By contrast, our Hermite snakes are defined explicitly by construction. They allow for extensive manipulation through a general user interface, although the segmentation process remains mostly automatic. The anchor points and tangent vectors can be manually tweaked in a user-friendly and precise manner. Such edit capabilities are of particular interest for complex image-analysis problems where feedback from the user might be required. Open- and closedsnake variants have been implemented as plugins for the opensource image-analysis software ImageJ [16]. They are freely available online for use by the bioimage-analysis community. ${ }^{1}$

There is an extensive literature describing various types of spline-snakes. Our contribution is first to provide a new snake model that holds some very particular properties differentiating it from existing active contours, but also to characterize the model in a comprehensive manner so as to highlight its practical scope. The paper is organized as follows: In Section II, we recall the general formulation of parametric snakes and specify our new snake. We describe the two active contour constructions (closed and open curves) that can be generated by our model and identify the key requirements that the

\footnotetext{
${ }^{1}$ http://bigwww.epfl.ch/algorithms/hsnakes
} 
Hermite snake should meet. In Section III, we translate the practical desiderata into a precise mathematical formulation. By relying on spline theory, we are able to study formally the aspects of our snake that make it well-suited for practical use. In Section IV, we discuss the features of the Hermite snake that allow for the reproduction of sharp discontinuities and the existence of orientation-based energy terms. Finally, in Section V, we study the validity and robustness of the proposed approach using synthetic images. We then illustrate its capabilities by comparing it against other spline-snake models. Finally, we provide results on real biological data in different experimental settings.

\section{The Hermite Spline Snake Model}

Throughout the paper, we denote 2-D vectors as $\mathbf{v}=\left(v_{1}, v_{2}\right)=\left[\begin{array}{l}v_{1} \\ v_{2}\end{array}\right]$. Let $\mathbf{r}=(x, y)$ be a 2-D curve on the image. It is described by the two Cartesian coordinate functions $x(t)$ and $y(t)$, with $t \in \mathbb{R}$ a continuous variable, that can be parameterized efficiently as the linear combination of the integer shifts of given basis functions weighted by a sequence of control points. In the case of spline snakes, the basis are constructed from a compactly supported generator $\phi$ and we denote the sequence of distinct control points as $\{\mathbf{c}[k]\}_{k \in \mathbb{Z}}$. The general expression of parametric spline snakes is therefore

$$
\mathbf{r}(t)=\left[\begin{array}{l}
x(t) \\
y(t)
\end{array}\right]=\sum_{k \in \mathbb{Z}} \mathbf{c}[k] \phi(t-k) .
$$

In our case, the generator of interest is composed of two cubic Hermite B-spline basis functions $\phi_{1}$ and $\phi_{2}$ that can be written in compact vector form as $\boldsymbol{\phi}=\left(\phi_{1}, \phi_{2}\right)$. Then, we denote the space generated by their integer shifts as $\{\boldsymbol{\phi}(\cdot-k)\}_{k \in \mathbb{Z}}$. The novel aspect of our construction comes from the fact that, for a given curve, the function $\phi_{1}$ is dedicated to the interpolation of point values, while the derivative of $\phi_{2}$ interpolates the tangent values. Both $\phi_{1}$ and $\phi_{2}$ are compactly supported in $[-1,1]$ and benefit from fast and stable interpolation methods [17]. The analytic expression of $\phi_{1}$ and $\phi_{2}$ as well as the mathematical formulation of their joint interpolation properties is discussed in Section III.

Parametric snakes are most commonly defined as closed curves (see [18] for a review), although models handling open curves for the segmentation of lines or boundaries also exist [15], [19]-[22]. Our Hermite-snake formulation is particularly well adapted to both scenarios. A closed curve can easily be constructed relying on periodized versions of the Hermite basis, in direct analogy with classical spline snakes [14]. The generation of open classical cubic splinesnakes, however, requires one to rely on virtual or invisible control points at the curve extremities. By contrast, our cubic Hermite snake allows for the imposition of natural conditions at the snake's ends through the control of its tangent vectors.

\section{A. Open Hermite Snake}

Open curves are specified by a sequence of $M$ anchor points and their associated tangents vectors, defined respectively for

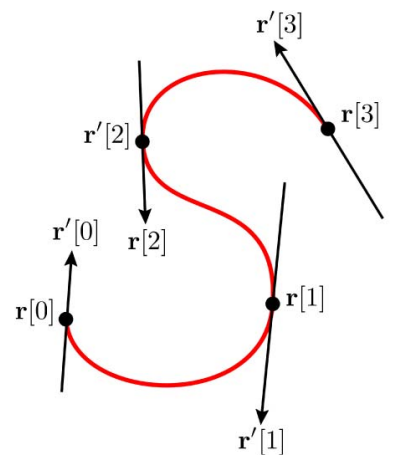

(a)

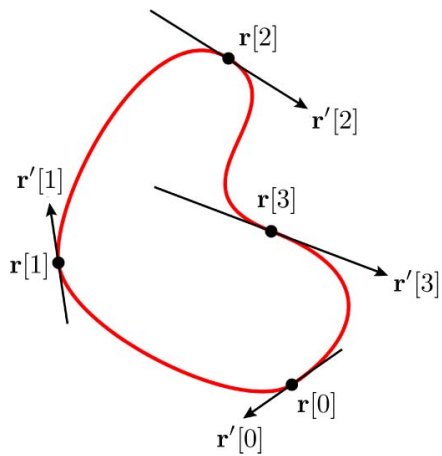

(b)
Fig. 1. (a) Open and (b) closed Hermite active-contour models. In both cases, the curve is represented by a set of control points and associated tangent vectors.

$k=0, \ldots, M-1$ as

$$
\begin{aligned}
\mathbf{r}[k] & =\left.\mathbf{r}(t)\right|_{t=\frac{k}{M-1}}, \\
\mathbf{r}^{\prime}[k] & =\left.\frac{\mathrm{d} \mathbf{r}}{\mathrm{d} t}(t)\right|_{t=\frac{k}{M-1}} .
\end{aligned}
$$

Due to the interpolation properties of the Hermite generators, control points and tangents directly correspond to the curve value at integer locations. The parametric representation of the open Hermite snake is then given by

$$
\begin{aligned}
\mathbf{r}_{\text {open }}(t)=\sum_{k=0}^{M-1}(\mathbf{r}[k] & \phi_{1}((M-1) t-k) \\
& \left.+\mathbf{r}^{\prime}[k] \phi_{2}((M-1) t-k)\right),
\end{aligned}
$$

where $t \in[0,1]$. An example curve is depicted in Figure 1a.

\section{B. Closed Hermite Snake}

Closed curves are obtained by periodizing the basis functions. In such case, the active contour is entirely defined by an $M$-periodic sequence of anchor points $\{\mathbf{r}[k]\}_{k \in \mathbb{Z}}$ and their tangent vectors $\left\{\mathbf{r}^{\prime}[k]\right\}_{k \in \mathbb{Z}}$. In analogy to the open-curve case,

$$
\begin{gathered}
\mathbf{r}[k]=\mathbf{r}[k+M]=\left.\mathbf{r}(t)\right|_{t=\frac{k}{M},} \\
\mathbf{r}^{\prime}[k]=\mathbf{r}^{\prime}[k+M]=\left.\frac{\mathrm{d} \mathbf{r}}{\mathrm{d} t}(t)\right|_{t=\frac{k}{M}} .
\end{gathered}
$$

We then rely on $M$-periodized versions of the generators

$$
\begin{aligned}
& \phi_{1, \mathrm{per}}(t)=\sum_{n=-\infty}^{\infty} \phi_{1}(t-M n) \\
& \phi_{2, \mathrm{per}}(t)=\sum_{n=-\infty}^{\infty} \phi_{2}(t-M n) .
\end{aligned}
$$

The continuously defined closed contour, which is hence 1 -periodic, is finally obtained as

$$
\begin{aligned}
\mathbf{r}_{\text {closed }}(t)=\sum_{k=0}^{M-1}\left(\mathbf{r}[k] \phi_{1, \operatorname{per}}(M t-k)\right. & \\
& \left.+\mathbf{r}^{\prime}[k] \phi_{2, \operatorname{per}}(M t-k)\right),
\end{aligned}
$$

where $t \in[0,1]$ (see Figure 1b). 
The positive integer $M$, corresponding to the number of control points/tangent pairs, regulates the flexibility of the resulting snake. Large values of $M$ allow for the reproduction of complex shapes, while small ones yield simple contours. It hence becomes possible to approximate any closed shape by considering an arbitrarily large amount of control points and associated tangents.

The model we propose is an extension of the cubic-spline snakes. In the absence of $\phi_{2}$, Equations (3) and (7) indeed reduce to the classical formulation (1) of parametric active contours. The novel aspect of our approach is the introduction of $\phi_{2}$ to enable control over the tangents. This construction grants several novel features discussed in more details in Section IV that are absent from the classical spline snakes.

The link between our Hermite-spline interpolation scheme and Bézier curves is explicitly formulated in Section III-F.

\section{Desired Properties of Hermite BASIS FUNCTIONS}

An active-contour model should satisfy several natural properties. Our goal hereafter is to specify our desiderata from a user point of view; these then motivate a formal analysis. The mathematical development and proof of each property has been placed in the Appendix for the interested reader.

For the sake of clarity, we consider through this section a 1D continuous curve $s$ instead of a $2 \mathrm{D}$ contour $\mathbf{r}$. As the first and second coordinate functions of $\mathbf{r}$ are parameterized separately, this does not induce any loss of generality in our statements.

\section{A. Characterization of Cubic Hermite Splines}

Cardinal Hermite-spline functions were introduced in [23]. Here, we focus on the cubic polynomial case. Note that a variety of Hermite schemes can be constructed, for instance based on exponential functions, as in [24] and [25].

The cubic-Hermite-spline function space is specified by the two generators

$$
\begin{aligned}
& \phi_{1}(t)=\left\{\begin{array}{lr}
(2|t|+1)(|t|-1)^{2} & \text { for } 0 \leq|t| \leq 1 \\
0 & \text { for } 1<|t|,
\end{array}\right. \\
& \phi_{2}(t)= \begin{cases}t(|t|-1)^{2} & \text { for } 0 \leq|t| \leq 1 \\
0 & \text { for } 1<|t| .\end{cases}
\end{aligned}
$$

Besides the restriction of the support to the interval $[-1,1]$, the fundamental property of this construction is that the generating functions $\phi_{1}, \phi_{2}$ and their derivatives $\phi_{1}^{\prime}, \phi_{2}^{\prime}$ satisfy the joint interpolation conditions

$$
\phi_{1}(k)=\delta[k], \quad \phi_{2}^{\prime}(k)=\delta[k], \quad \phi_{1}^{\prime}(k)=0, \quad \phi_{2}(k)=0,
$$

for all $k \in \mathbb{Z}$. The functions and their first derivative are depicted in Figure 2, where the interpolation properties can easily be observed. Note that $\phi_{1}$ and $\phi_{2}$ are cubic polynomials in each interval $[k, k+1)$ for $k=-1,0$ and, by extension, $k \in \mathbb{Z}$. They are differentiable with continuous derivatives at the integer knots points $t=k$. The functions $\phi_{1}$ and $\phi_{2}$ are deeply intricated: they solve together the interpolation problem of the function and its derivative at the integers as exposed
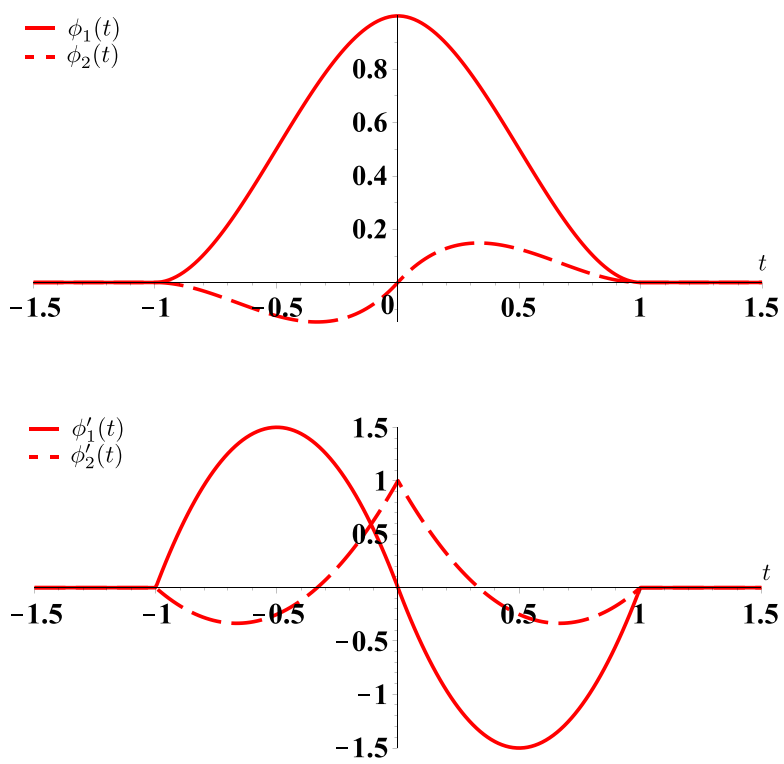

Fig. 2. Generators $\phi_{1}$ and $\phi_{2}$ for the cubic Hermite splines. The two functions and their derivatives are vanishing at the integers with the exception of $\phi_{1}(0)=1$ and $\phi_{2}^{\prime}(0)=1$ (interpolation conditions). Their support is $[-1,1]$.

in [23]. All the fundamental properties of Hermite basis functions exposed in Section III are based on this intrication.

Linear combinations of the integer shifts of these generators generate functions that are $C^{1}$-continuous piecewise-cubic polynomials with knots at integer locations. Such functions can also be interpreted as cubic splines with double knots on the integers. The effect of the double knot is to reduce the degree of continuity $\left(C^{2}\right)$ of the classical cardinal cubic splines by one, hence allowing sharp corners.

The space of functions generated by the Hermite spline scheme is defined as

$$
\left\{s(t)=\sum_{k \in \mathbb{Z}}\left[\begin{array}{c}
s[k] \\
s^{\prime}[k]
\end{array}\right]^{T} \phi(t-k): s[k], s^{\prime}[k] \in \ell_{2}(\mathbb{Z})\right\}
$$

with the vector $\phi=\left(\phi_{1}, \phi_{2}\right)$. Due to the Hermite interpolation conditions, the expansion coefficients $s[k]$ and $s^{\prime}[k]$ in (11) coincide with $s(k), s^{\prime}(k)$, the samples of the function and its first derivative on the integer grid.

\section{B. Connection With Classical Cubic and Quadratic Splines}

In order to better understand the properties of the polynomial Hermite-spline scheme, we here highlight its connection with the classical cubic and quadratic splines. Our way of establishing this link is to compute the Fourier transforms of the Hermite-spline generators. We denote the Fourier transform of a function $f$ as $\widehat{f}$. This yields

$$
\widehat{\phi}(\omega)=\left[\begin{array}{l}
\widehat{\phi}_{1}(\omega) \\
\widehat{\phi}_{2}(\omega)
\end{array}\right]=\left[\begin{array}{c}
-\frac{12(\omega \sin \omega+2 \cos \omega-2)}{\omega^{4}} \\
-\frac{4 \mathrm{j}(2 \omega-3 \sin \omega+\omega \cos \omega)}{\omega^{4}}
\end{array}\right] .
$$

These equations can be rewritten in matrix-vector form as

$$
\widehat{\phi}(\omega)=\widehat{\mathbf{R}}\left(\mathrm{e}^{\mathrm{j} \omega}\right) \widehat{\boldsymbol{\rho}}(\omega),
$$


with

$$
\widehat{\boldsymbol{\rho}}(\omega)=\left[\begin{array}{c}
\widehat{\rho}_{1}(\omega) \\
\widehat{\rho_{2}}(\omega)
\end{array}\right]=\left[\begin{array}{c}
\frac{1}{(\mathrm{j} \omega)^{4}} \\
\frac{1}{(\mathrm{j} \omega)^{3}}
\end{array}\right]
$$

and

$$
\widehat{\mathbf{R}}\left(\mathrm{e}^{\mathrm{j} \omega}\right)=\left[\begin{array}{cc}
24-12 \mathrm{e}^{-\mathrm{j} \omega}-12 \mathrm{e}^{\mathrm{j} \omega} & -6 \mathrm{e}^{-\mathrm{j} \omega}+6 \mathrm{e}^{\mathrm{j} \omega} \\
-6 \mathrm{e}^{-\mathrm{j} \omega}+6 \mathrm{e}^{\mathrm{j} \omega} & -8-2 \mathrm{e}^{-\mathrm{j} \omega}-2 \mathrm{e}^{\mathrm{j} \omega}
\end{array}\right] .
$$

The vector $\boldsymbol{\rho}=\left(\rho_{1}, \rho_{2}\right)$, the inverse Fourier transform of $\widehat{\boldsymbol{\rho}}$, contains the Green's functions of the operators $L_{1}=\frac{\mathrm{d}^{4}}{\mathrm{~d} t^{4}}$ and $\mathrm{L}_{2}=\frac{\mathrm{d}^{3}}{\mathrm{~d} t^{3}}$ which generate the classical cubic and quadratic splines, respectively. From this, we show in Appendix A that the cubic and quadratic B-splines can be expressed in terms of $\phi_{1}$ and $\phi_{2}$. Thus, they are included in the family of Hermite splines.

\section{Uniqueness and Stability of the Representation}

A given parametric curve should be uniquely defined by its anchor points and control tangents, and numerical stability of the interpolation process should be ensured. These two properties are summarized in the so-called Riesz-basis condition for the generator $\phi$. By definition (see for instance [26, Sec. 6.2.3]), the vector function $\phi=\left(\phi_{1}, \phi_{2}\right)$ generates a Riesz basis if and only if there exist two constants $0<A \leq B<+\infty$ such that

$$
A\|\mathbf{a}\|_{\ell_{2}} \leq\left\|\sum_{k \in \mathbb{Z}} \mathbf{a}[k]^{T} \boldsymbol{\phi}(\cdot-k)\right\|_{L_{2}} \leq B\|\mathbf{a}\|_{\ell_{2}},
$$

for all $\mathbf{a}[k]=\left(s[k], s^{\prime}[k]\right), s[k], s^{\prime}[k] \in \ell_{2}(\mathbb{Z})$, where $s[k]$ and $s^{\prime}[k]$ are the expansion coefficients of $s(\cdot)$ in the Hermite basis. In Appendix B, we show by relying on Fourier domain results that this condition is verified for the Hermite splines.

\section{Precise Reproduction of Curves}

The difference between an arbitrary reference curve and its cubic Hermite-spline model helps us define the quality of approximation of our active contour. In particular, we want to know at which rate the approximation error decreases when approximating any function $f \in L_{2}(\mathbb{R})$ with $\boldsymbol{\phi}=\left(\phi_{1}, \phi_{2}\right)$ and its integer shifts.

As shown in Appendix A, one can reproduce both quadratic and cubic polynomial splines using the cubic Hermite splines (see (44) and (46)). It means in particular that the asymptotic approximation properties of the Hermite spline space are at least as good as those of the popular space of cubic splines.

It can actually be shown that the Hermite spline space corresponds to the direct sum of the cubic and quadratic spline spaces. It is then possible to apply the multifunction analysis from [27], in particular Theorem 1, to the case of the Hermite basis functions. By doing so, one can demonstrate that the cubic Hermite splines have the same asymptotic approximation order as the cubic polynomial splines.

\section{E. Smoothness Properties}

Many parametric active contours come with inherent smoothness properties [28], [29]. The associated snakes therefore do not need to rely on additional energy terms (called internal energies) to ensure smoothness of the contour. This is of particular interest as internal energy terms are observed to play a major role in practical applications [3], [30].

The smoothness properties of our Hermite splines are related to Theorem 1, whose proof is given in Appendix C.

Theorem 1: Let $s[k], s^{\prime}[k] \in \ell_{2}(\mathbb{Z})$. Among all possible functions $f(t), t \in \mathbb{R}$ with $f, f^{\prime}, f^{\prime \prime} \in L_{2}(\mathbb{R})$ the optimal one that minimizes

$$
\left\|f^{\prime \prime}\right\|_{L_{2}}
$$

such that $s[k]=f(k)$ and $s^{\prime}[k]=f^{\prime}(k)$ for $k \in \mathbb{Z}$ is the Hermite interpolator $s$ defined as

$$
s(t)=\sum_{k \in \mathbb{Z}}\left(s[k] \phi_{1}(t-k)+s^{\prime}[k] \phi_{2}(t-k)\right) .
$$

Cubic Hermite splines are therefore optimal in the sense that they minimize $\left\|f^{\prime \prime}\right\|_{L_{2}}$, which is a good approximation of the curvature [13]. It therefore holds the potential to eliminate the need for an explicit internal energy term. If desired, low curvature could therefore be guaranteed under some mild conditions on the parametric curve in a similar fashion to cubic spline snakes [13].

Our result is closely related to the well-known minimumnorm property of cubic splines which states that, given a set of nodes in an interval $[a, b]$, the cubic-spline interpolator minimizes $\int_{a}^{b}\left|f^{\prime \prime}(t)\right|^{2} \mathrm{~d} t$ among all interpolating functions $f$, as shown with some variations in [31, Th. 3.1.1] and [12, Th. 1].

\section{F. Connection With Bézier Representations}

The presence of explicit tangents in our representation is reminiscent of the Bézier curves which are popular in computer graphics. We discuss now how these two representations relate to one another through the use of Bernstein polynomials; in particular, we show how to express Hermite splines in terms of Bézier curves.

Let us consider the task of computing

$$
s(t)=\sum_{k \in \mathbb{Z}}\left(s[k] \phi_{1}(t-k)+s^{\prime}[k] \phi_{2}(t-k)\right)
$$

for $t \in[n, n+1)$ and some $n \in \mathbb{Z}$. Defining $u=t-n \in[0,1)$, we simplify the expansion as

$$
\begin{aligned}
s(n+u)= & s[n] \phi_{1}(u)+s^{\prime}[n] \phi_{2}(u)+s[n+1] \phi_{1}(u-1) \\
& +s^{\prime}[n+1] \phi_{2}(u-1)
\end{aligned}
$$

by retaining only the four Hermite basis functions that do not vanish within the unit interval $[0,1)$. They are shown on the top plot of Figure 3. Since $s(n+u)$ for $u \in[0,1)$ is a cubic polynomial by construction, we may express it using the Bernstein polynomial basis which is also a basis of the Bézier curves. The four Bernstein cubic polynomials are

$$
\begin{aligned}
& b_{0,3}(u)=(1-u)^{3}, \quad b_{1,3}(u)=3 u(1-u)^{2}, \\
& b_{2,3}(u)=3 u^{2}(1-u), \quad b_{3,3}(u)=u^{3} .
\end{aligned}
$$

The conversion between the two types of representations is 

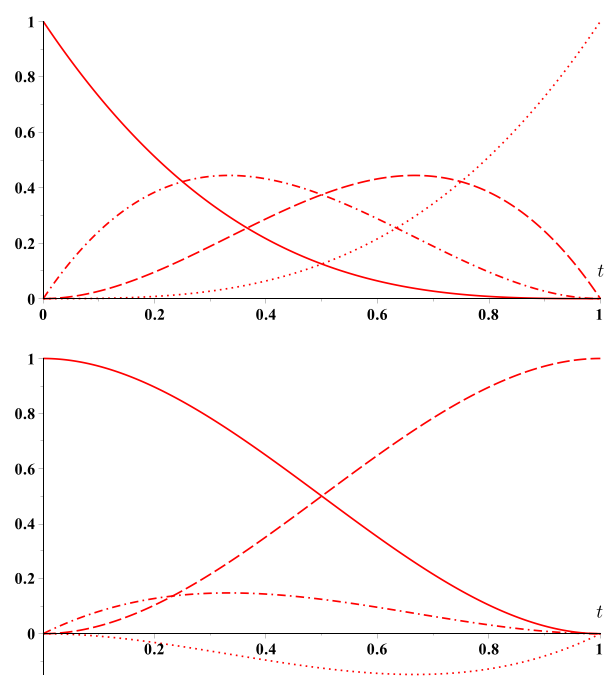

Fig. 3. Comparison of different polynomial bases on the unit interval $[0,1)$. (Top) Restriction of the cubic Hermite basis on $[0,1)$. (Bottom) The four cubic Bernstein polynomials.

$$
\left[\begin{array}{c}
\phi_{1}(u) \\
\phi_{2}(u) \\
\phi_{1}(u-1) \\
\phi_{2}(u-1)
\end{array}\right]=\left[\begin{array}{rrrr}
1 & 1 & 0 & 0 \\
0 & +\frac{1}{3} & 0 & 0 \\
0 & 0 & 1 & 1 \\
0 & 0 & -\frac{1}{3} & 0
\end{array}\right] \cdot\left[\begin{array}{c}
b_{0,3}(u) \\
b_{1,3}(u) \\
b_{2,3}(u) \\
b_{3,3}(u)
\end{array}\right]
$$

By plugging these expressions into (19), the Bézier representation of $s(n+u)$ is

$$
s(n+u)=p_{0} b_{0,3}(u)+p_{1} b_{1,3}(u)+p_{2} b_{2,3}(u)+p_{3} b_{3,3}(u),
$$

with control values

$$
\begin{aligned}
& p_{0}=s[n], \quad p_{1}=s[n]+\frac{1}{3} s^{\prime}[n], \\
& p_{2}=s[n+1]-\frac{1}{3} s^{\prime}[n+1], \quad p_{3}=s[n+1] .
\end{aligned}
$$

From there, the extension to parametric curves in $\mathbb{R}^{2}$, which is our principal interest for active-contours applications, is straightforward. The 2-D curve given by

$$
\mathbf{r}(t)=\left[\begin{array}{l}
x(t) \\
y(t)
\end{array}\right]=\sum_{k \in \mathbb{Z}}\left(\mathbf{r}[k] \phi_{1}(t-k)+\mathbf{r}^{\prime}[k] \phi_{2}(t-k)\right),
$$

where $\mathbf{r}^{\prime}(t)=\left(\frac{\mathrm{d} x(t)}{\mathrm{d} t}, \frac{\mathrm{d} y(t)}{\mathrm{d} t}\right)$ is the tangent vector of the curve, can be expressed in Bernstein representation for each interval $[n, n+1)$ as

$$
\mathbf{r}(n+u)=\mathbf{p}_{0} b_{0,3}(u)+\mathbf{p}_{1} b_{1,3}(u)+\mathbf{p}_{2} b_{2,3}(u)+\mathbf{p}_{3} b_{3,3}(u)
$$

with control points

$$
\begin{aligned}
& \mathbf{p}_{0}=\mathbf{r}[n], \quad \mathbf{p}_{1}=\mathbf{r}[n]+\frac{1}{3} \mathbf{r}^{\prime}[n], \\
& \mathbf{p}_{2}=\mathbf{r}[n+1]-\frac{1}{3} \mathbf{r}^{\prime}[n+1], \quad \mathbf{p}_{3}=\mathbf{r}[n+1] .
\end{aligned}
$$

These four points define the control polygon that outlines the shape of the underlying curve segment. Specifically, $\mathbf{p}_{0}=\mathbf{r}[n]$ and $\mathbf{p}_{3}=\mathbf{r}[n+1]$ are the start and end points, while the corresponding tangent directions are indicated by the vectors $\mathbf{p}_{1}-\mathbf{p}_{0}=\mathbf{r}^{\prime}[n]$ and $\mathbf{p}_{3}-\mathbf{p}_{2}=\mathbf{r}^{\prime}[n+1]$, respectively. The fact that the Bernstein basis is positive also ensures that the curve segment is enclosed in the convex hull of the control polygon, which is not the case for the Hermite basis.

The Hermite snake representation can therefore be easily converted to a Bézier curve, but the converse is not true in general. Indeed, by construction, Bézier curves can have different left and right tangents. A Bézier curve therefore has more degrees of freedom, but is also less regular. The increased smoothness granted by the Hermite snake representation is especially useful for practical applications, as it implies that less parameters have to be optimized and one benefits from additional intrinsic regularity.

\section{Applicative Aspects of the Hermite Snake}

We now present the particular features of the associated Hermite snake. The two main novel aspects brought by our Hermite construction are the ability to reproduce corners easily and the possibility to design directional energy functionals. Additional aspects are related to implementation and user interactions.

\section{A. Generation of Corners}

An important feature of the Hermite snake is its ability to reproduce corners with only one control point, something that is not achievable with classical spline snakes.

To better understand how the Hermite-snake model enables the generation of corners, we start by considering the properties that define this feature. A necessary condition for introducing a corner at curvilinear coordinate $t_{0}$ is to set the derivatives of the coordinate functions to

$$
x^{\prime}\left(t_{0}\right)=y^{\prime}\left(t_{0}\right)=0 .
$$

What we call corners are often referred to as singular points [32].

It can be shown that in the cubic- and exponential-spline snake cases, (27) can only be satisfied when all control points lie on a straight line, which we refer to as a flat corner. This situation is obviously of limited practical interest. The Hermite snake, however, allows one to enforce (27) by setting the tangent controls to zero at any arbitrary control point. When all tangent controls in a Hermite snake are set to zero (i.e., $s^{\prime}(k)=0$ for all $k$ ), it is not hard to show that $\frac{y(t)}{x(t)}$ is constant in $[k, k+1)$ for every $k$ on the curve. In such a situation, we are hence back to linear interpolation and control points are linked by straight lines. Setting a nonnull tangent vector introduces smoothness on the curve at the corresponding control point, as shown in Figure 4. Conversely, in a curve composed of mostly non-zero tangent vectors, setting one of them to zero results in a local roundish corner as depicted in Figure 5.

As a more precise explanation, two situations allow creating a true, non-flat corner. The local slopes in each coordinate functions around the corner point can either be different or equal to zero. The first situation with unequal local slopes around the corner point implies that the second derivativeof either coordinate function-contains a discontinuity. Cubic 

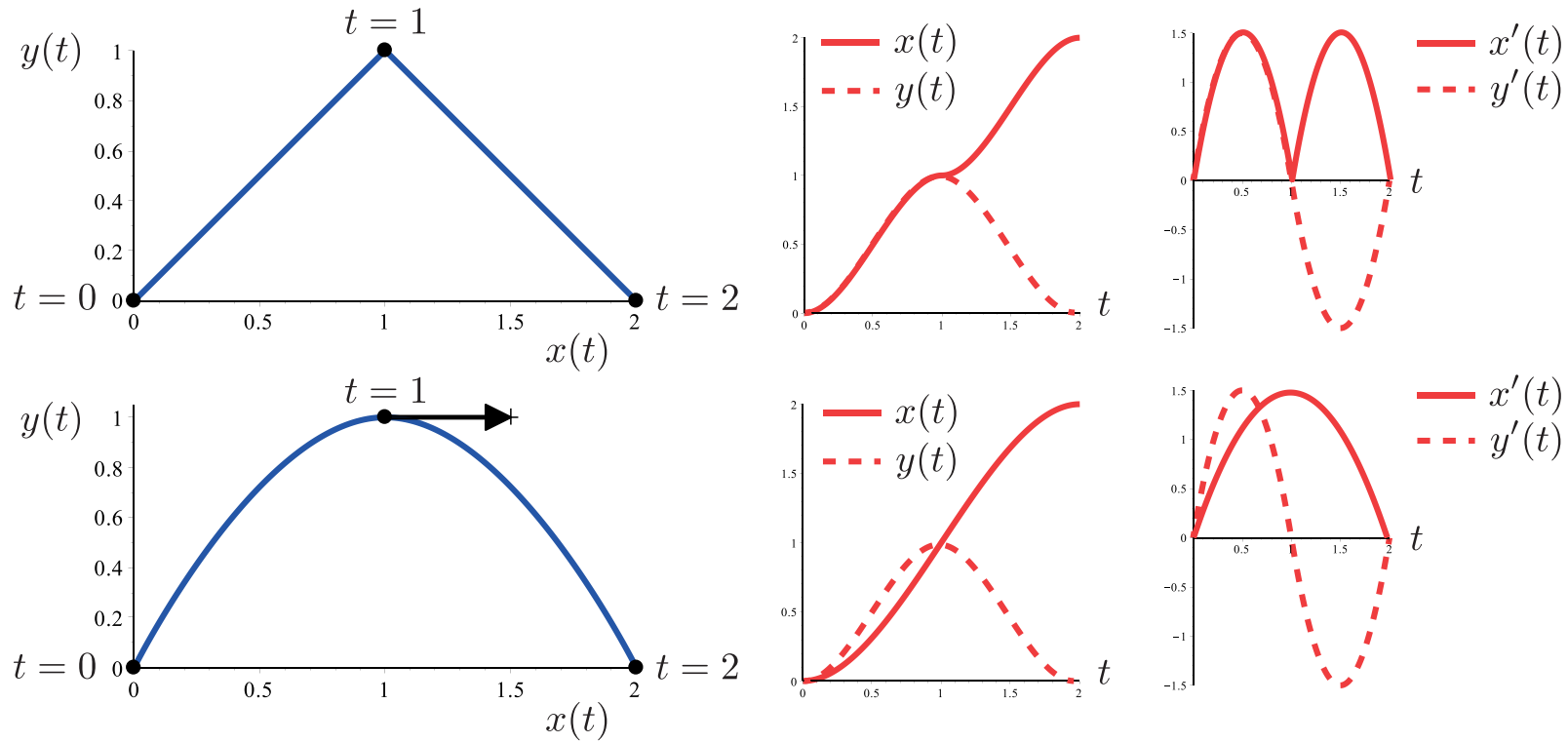

Fig. 4. Generation of corners with the Hermite snake. Two 2-D curves are depicted as solid lines along with a plot of their coordinate functions and their derivatives. The curves are parameterized by three control points (solid dots) and associated tangent vectors (arrows, set to vanish at $\mathbf{r}(0)$ and $\mathbf{r}(2)$ ). The control points of the two curves are identical, except for the tangent at $\mathbf{r}(1)$. When set to zero, it creates a discontinuity in $x^{\prime \prime}$ and yields a sharp corner. When it does not vanish, the discontinuity disappears and a smooth curve is obtained.

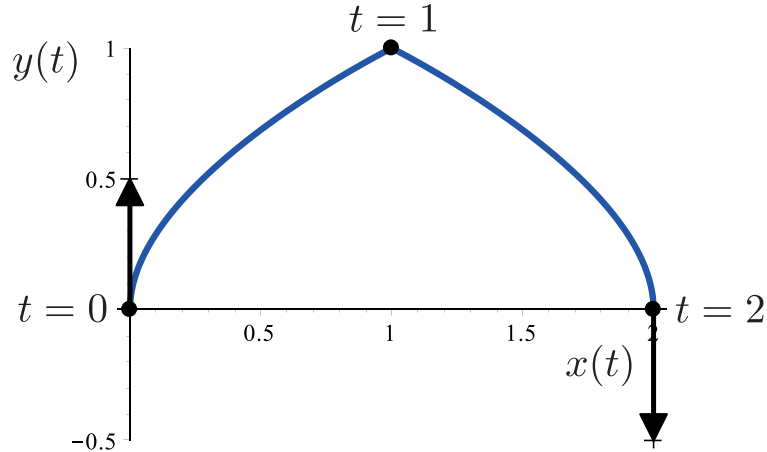

Fig. 5. Generation of roundish corners with the Hermite snake. Setting $x^{\prime}(1)=y^{\prime}(1)=0$ creates a corner. Although the surrounding control points have non zero derivatives (represented by arrows), the local behavior around the corner is conserved and yields a sharp discontinuity at $\mathbf{r}(1)$.

and exponential splines, and associated spline-snake curves, are $C^{2}$ by construction at the joining points and hence have continuous second derivatives. Hermite-spline snakes, on the contrary, are $C^{1}$ at the joining points and therefore allow for discontinuous second derivatives. It is still possible to generate corners with cubic or exponential splines through the second scenario, where slopes around the corner point are vanishing. However, it implies for several control points to accumulate at the same location on the 2-D plane. This corresponds to the introduction of multiple knots in the spline curve, and thus does not fit the spline-snake formalism relying on a sequence of distinct control points. ${ }^{2}$

\section{B. Directional Energy Functionals}

The evolution of active contours is driven by the optimization of a cost functional referred to as snake energy. Many

${ }^{2}$ The curve $\mathbf{r}(t)=\left(t^{3}, t^{2}\right)$ is an example of this situation, with a corner at $\mathbf{r}(0)$. energy terms have been proposed in the literature [13], [33]. The specificity of the image-analysis problem usually drives the choice of a given energy function, as it dictates the quality of the result.

Relying on a spline formulation, the Hermite snake is versatile enough to handle all traditional energy functionals used with classical parametric and point-based snakes. The novelty of our model lies in the direct expression of the tangents in the snake formulation. This enables the definition of novel energy terms imposing constraints on the local orientation of the contour.

Given a snake curve $S$ parameterized by $\mathbf{r}:[0,1] \mapsto S$, we define the continuous directional snake energy functional as

$$
E_{\text {directional }}=-\frac{1}{L} \int_{S} \mid\left\langle\theta, \frac{\mathbf{r}^{\prime}}{\left\|\mathbf{r}^{\prime}\right\|}\right\rangle \rho(\mathbf{r}) \mathrm{d} \mathbf{r},
$$

where $\theta$ and $\rho$ are some orientation and amplitude information. The 2-D vector functions $\mathbf{r}=(x, y)$ and $\mathbf{r}^{\prime}=\left(x^{\prime}, y^{\prime}\right)$ correspond to the position and derivative of the curve, respectively. The absolute value of the inner product between $\boldsymbol{\theta}$ and the normalized $\mathbf{r}^{\prime}$, which appears as the first term of the integrand, is large when the tangents of the contour are locally aligned with the orientation given by $\theta$. The term $\rho$ is a weight that favors locations corresponding to a large amplitude of some chosen image-based feature. In order to obtain a dimensionless energy, the line integral is finally normalized by the length $L$ of the curve. The whole expression is set with a minus sign since the snake optimization is usually defined as a minimization process.

The directional energy functional differs from classical image-based energies by the addition of the term that enforces $\theta$ and $\mathbf{r}^{\prime}$ to have the same orientation. The ability to constrain the behavior of the derivative of the snake through the tangent vectors increases the overall robustness of the segmentation. 
In cases where several patterns of interest are in close contact or when dealing with object with complex shapes, magnitude information might not be sufficient to properly segment the contour.

The proposed directional energy functional (28) is general enough to accommodate any image-wide functions $\theta$ and $\rho$ providing orientation and amplitude information, respectively. We here propose two different cases. We denote by $f$ the input image.

1) Image Gradient: The orientation and magnitude functions are given by the classical 2D gradient orientation and amplitude

$$
\begin{aligned}
& \theta(\mathbf{x})=\arctan \left(\frac{\frac{\partial f(\mathbf{x})}{\partial y}}{\frac{\partial f(\mathbf{x})}{\partial x}}\right), \\
& \rho(\mathbf{x})=\sqrt{\left(\frac{\partial f(\mathbf{x})}{\partial x}\right)^{2}+\left(\frac{\partial f(\mathbf{x})}{\partial y}\right)^{2}} .
\end{aligned}
$$

The resulting snake energy will tend to place the snake contour in regions of high gradient amplitude (i.e., edges) and to align its tangent vectors with the local orientation of the gradient. As $\theta$ tends to be noisy, this choice might not be robust in practice.

2) Steerable Filters: Steerable filters offer a more refined version of the directional energy functional. From [34], orientation and magnitude can be defined as

$$
\begin{aligned}
& \boldsymbol{\theta}(\mathbf{x})=\underset{\vartheta}{\operatorname{argmax}}\left(\left(f * h\left(\mathbf{R}_{\vartheta} \cdot\right)\right)(\mathbf{x})\right), \\
& \rho(\mathbf{x})=\left(f * h\left(\mathbf{R}_{\boldsymbol{\theta}} \cdot\right)\right)(\mathbf{x}),
\end{aligned}
$$

where $h$ is the detection template given by

$$
h(\mathbf{x})=\sum_{k=0}^{N} \sum_{i=0}^{k} a_{k, i} \frac{\partial^{k-i}}{\partial x^{k-i}} \frac{\partial^{i}}{\partial y^{i}} g(\mathbf{x}) .
$$

In (33), $g$ is a Gaussian window and $N$ is the order of the detector. The features detected by $h$ can be modified by acting on $N$. An odd $N$ yields edge detectors while an even $N$ detects ridges. In addition, filters built with large values of $N$ have a high SNR and good localization capabilities, the tradeoff being the computational cost [34]. Finally, the orientation information given by steerable filters is more precise than that obtained using the Canny edge and Hessian ridge detectors.

\section{Snake Optimization}

For the sake of efficiency, the values of the Hermite basis functions $\phi_{1}$ and $\phi_{2}$ are precomputed and stored in lookup tables. This allows for a real-time response when interacting with the anchor points and tangent vectors of the snake. The subsequent automated optimization is carried out in an efficient way relying on Powell-like line-search methods [35]. This algorithm converges quadratically and requires the computation of the derivatives of the energy function with respect to the parameters (i.e., the coefficients of the Hermite spline). Its speed can sometimes be increased further by deriving a closed-form expression of the derivatives of the snake energy. However, while it has been shown in [14] that having an analytical expression for the energy gradient is especially useful when the number of parameters becomes large, our snake is defined by a small number of parameters ( $M$ anchor points plus $M$ tangent vectors in 2D). Therefore, the centered finite differences approach is satisfying in most practical cases.

\section{User Interaction}

Open and closed snakes based on the Hermite-spline framework have been implemented as open-source plugins for the multiplatform software ImageJ [16]. They are freely available on the Biomedical Imaging Group website. ${ }^{3}$ Note that these plugins are more complete and hold substantial changes compared to the one published in [11].

Our Hermite active-contour model facilitates interaction with the user in several ways. First, it relies on a smaller number of control points than many other parametric snake models. Then, since its construction involves basis functions of small support, the snake is only locally affected by structural changes. This implies that modifications of one control point merely affect the curve in a small local neighborhood. Finally, due to the interpolation properties of a Hermite spline, anchor points truly lie on the actual contour on the snake.

We take advantage of this framework to provide an intuitive user interface in which the position and shape of the snake can be manually edited through manipulation of the control points and associated handles that correspond to their tangents. To this, we added the possibility to manually freeze control points and/or tangent handles at specific locations (corners, typically). When launching the automated optimization, the frozen points are conserved while the rest of the curve automatically adapts to follow the object of interest. As a result, our method can be deployed interactively by combining steps of snake initialization, optimization, and manual correction.

\section{E. Implementation Details}

The Hermite snake plugin is implemented as a direct translation from the theory presented in Section II. More precisely, snake curves are constructed making direct use of equations (3) (for the open curve case) and (7) (for the closed curve case). Since Hermite snakes are continuously defined in our model and practical implementations lie in the digital world, we rely on a discretization of the continuous parameter $t$ at a fixed sampling rate. To speed up execution time, samples of the basis functions $\phi_{1}$ and $\phi_{2}$ are also precomputed and stored in lookup tables.

\section{VALIDATION AND EXPERIMENTS}

In this section, we carry out the following experiments to demonstrate the practical usefulness of the Hermite snake. We proceed in three steps. First, we compare the Hermite snake against state-of-the-art parametric spline snakes. To do so, we study both the introduction of sharp corners in the snake curve and the result of segmentation in the presence and the absence of a directional energy functional. Second, we quantitatively study the robustness of our model to noise, change in

\footnotetext{
${ }^{3}$ http://bigwww.epfl.ch/algorithms/hsnakes/
} 
the number of control points, and initial conditions relying on Jaccard indices. Finally, we illustrate the use of the Hermite snake in practice with four different sets of bioimages acquired using brighfield, phase-contrast, differential-interferencecontrast (DIC), and scanning-electron microscopy. In the first two parts, we rely on synthetic test images.

For all experimental results presented in this section, the Hermite snake is solely driven by a directional energy functional of the form (28), where the orientation and magnitude information is given by a properly tuned steerable filter. Even in the closed-curve case, we do not include any form of region energy so as to be able to optimize both the open and closed versions of the snake using the same functional. In the literature, the snake energy is usually split into an external energy term, which contains all data-driven functionals, and an internal energy term encompassing the regularization energies. In the present case, snake optimization is therefore only carried out relying on an external energy term, which is equal to $E_{\text {directional. }}$

For the quantitative evaluation of the snake performance, we rely on the Jaccard index $J \in[0,1]$ defined as

$$
J=\frac{\left|S \cap S_{\text {ref }}\right|}{\left|S \cup S_{\text {ref }}\right|},
$$

where $S_{\text {ref }}$ corresponds to the ground-truth region and $S$ to the region segmented by the snake. It therefore corresponds to the percentage of similarity between the two regions computed with a pixelwise discretization, 1 being perfect segmentation. Note that because of the discretization, a Jaccard index of 1 is almost never attained and 0.99 can be considered as accurate segmentation.

\section{A. Comparison With Existing Approaches}

State-of-the-art spline snakes such as the cubic and exponential ones [14] adhere to the same principles than the Hermite snake, and provide therefore the most relevant competing methods. Here, we show how the two novel features granted by the explicit introduction of tangents in the Hermite-snake model, namely, the ability to design directional energy functionals and to reproduce corners, are advantageous over existing models.

1) Reproduction of Corners: Cubic- and exponential-spline snakes generate curves that are smooth by design. Sharp angles can be approximated but require a large number of distinct control points, as shown in Figures $6 \mathrm{a}$ and $6 \mathrm{~b}$. Conversely, the Hermite snake is able to introduce sharp corners in curves with only one control point, as illustrated in Figure 6c. In this Figure, the cubic and exponential snakes are constructed with 9 control points and yield a poor approximation of a sharp tip. With 4 control points and associated tangent vectors, the Hermite snake is perfectly able to recreate the tip of a droplike shape in Figure 6. As the stability of the optimization process depends on the number of parameters, the Hermite snake is less likely to diverge.

2) Directional Energy Functionals: The addition of orientation information in the snake energy enhances the robustness of the segmentation. It is of particular importance in the two following situations: when the contour of the object of interest

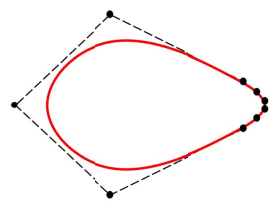

(a)

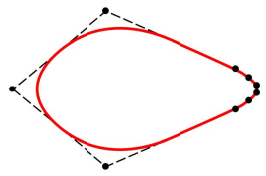

(b)

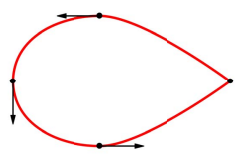

(c)
Fig. 6. Generation of sharp corners using (a) traditional cubic-spline snake, (b) exponential-spline snake, and (c) Hermite-spline snake.

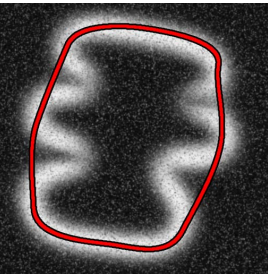

(a)

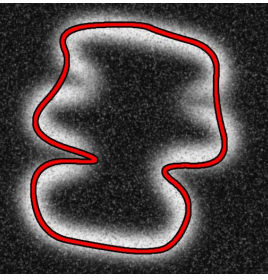

(b)

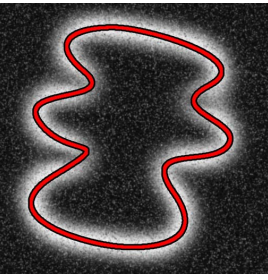

(c)
Fig. 7. Usefulness of orientation in the closed-curve case.(a) Snake initialization for (b) and (c). (b) Segmentation result after optimizing a 16-point exponential-spline snake with the ridge-based energy (35). (c) Segmentation result after optimizing an 8-point Hermite snake with (28).

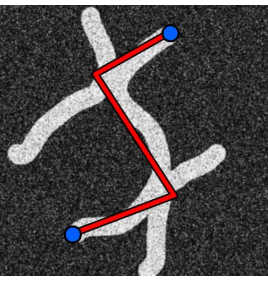

(a)

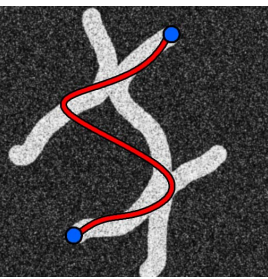

(b)

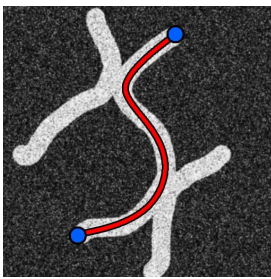

(c)
Fig. 8. Usefulness of orientation in the open-curve case.(a) Snake initialization for (b) and (c). (b) Segmentation result after optimizing an 8-point cubic-spline snake with the ridge-based energy (35). (c) Segmentation result after optimizing a 4-point Hermite snake with (28). Frozen points are depicted as disks.

contains rapid changes of orientation or has circonvolutions, and when nearby objects are likely to attract the snake and divert it. In both cases, information ignoring orientation might be unable to provide a correct segmentation.

To illustrate this, we generated synthetic images representative of these two situations. Using the same initial curve, we optimized a cubic, an exponential [14], and a Hermite snake, and compared segmentation results. We carried out all optimizations using a steerable filter designed to detect ridges constructed following [34]. In the Hermite case, we took advantage of (28). For the other snakes, we used the magnitude information

$$
E_{\text {magnitude }}=-\frac{1}{L} \int_{S} \rho(\mathbf{r}) \mathrm{d} \mathbf{r},
$$

Traditional spline snakes do not include tangents in their parameterization. To perform a fair comparison and be consistent in the number of parameters, we therefore endowed such snakes with twice as many control points as the Hermite snake. Results are shown in Figure 7 for the closed-curve case and in Figure 8 for the open-curve case. One can observe that the addition of orientation makes the snake more robust to the presence of circonvolutions and of nearby objects. 


\section{B. Model Validation}

1) Degrees of Freedom: The number $M$ of control points is the main parameter of the Hermite-snake model. The choice of $M$ should be guided by the particular segmentation task being considered, keeping in mind that larger values of $M$ grant flexibility to the snake and hence allow the reproduction of more complex shapes, but also enlarge the search space of the optimizer, which is therefore more likely to diverge or reach local minima. A few control points are therefore sufficient to segment smooth shapes well, while snakes with large $M$ tend to get entangled. For rougher, more complex contours, snakes with more control points and hence more degrees of freedom are required.

Since our purpose was to evaluate the adequacy of our parametric snake model, we fixed the number of control points for each experiment. There are also ways to choose the number of control points automatically. For instance, one can apply a multiresolution strategy and perform several rounds of optimization starting from a snake with very few control points, optimizing it, resampling the resulting curve with more control points, further optimizing, and iterating in this way until the snake energy at convergence stops decreasing. We did not study such approaches here to avoid overloading the paper.

2) Dependence on the Initial Conditions: Another important aspect is the initial snake curve from which the automated optimization process is started. A rough sketch should be sufficient since the optimization process takes care of precisely adapting it to the object boundary. Circular or oval shapes for closed snakes and broken lines for open ones are common initial contours. Another important aspect to consider is that the nature of the chosen energy term influences the convergence of snakes.

3) Robustness to Noise: We investigated the robustness of the Hermite snake to noise in the image as a function of the number of coefficients $M$. We created two synthetic images, one for the closed- and one for the open-curve case. We generated 100 realizations of these images in different PSNR conditions by adding a mixture of Gaussian and Poisson noises. Median Jaccard indices for each experimental setting are presented in Table I. Snake initializations are overlaid in the thumbnails which depict the noise-corrupted images. In the closed-curve case, one observes that the performance of the snake degrades faster for large number of control points as the amount of noise increases. This can be explained by the presence of local minima that are induced by noise for snakes with large $M$. In the open-curve case, the decrease in performance for low PSNR is more gradual due to the imposition of curve extremities, which reduces the risk of entanglement for large values of $M$.

4) Considerations on Execution Time: The automated optimization of the Hermite snake from a given initial curve runs in real time. The optimization process can either be clamped to a given number of iterations or can be left running until a minimum is found. The time required for optimization is affected by the number of control points. The change in execution time is however hardly noticeable for a reasonable range of control point values (i.e., $M=2, \ldots, 10$ ). The number of manual clicks required to get an appropriate segmentation depends both on the initial shape and on the eventual corrections to perform after automated optimization. It remains significantly lower than manual segmentation.

\section{Segmentation of Bioimages}

We here provide insights into the capabilities of the Hermite snake to segment bioimages. As we do not possess groundtruth information for the images presented in this section, we only rely on qualitative assessments for the quality of segmentation. For comparison purpose, we also provide results obtained using classical spline snakes. We rely on cubic spline snakes for the open curve case, and on exponential spline snakes [14] for the closed curve case. In all experiments, we use the same initialization and then let the snakes evolve automatically until convergence. To account for the fact that Hermite snakes have twice more parameters per control points, we doubled the number of control points for the classical snakes.

1) Detection of Medial Axis in Nematodes: The task in this experiment is to get a good approximation of the medial axis of the purely enteric worms Heligmosomoides polygyrus bakeri (H. bakeri). ${ }^{4}$ The unstained worms appear as translucent with uneven interior. Depending on experimental conditions, the worms are either "clean" or get cluttered by immune cells. By adapting the number of control points to the complexity of the worm shape and initializing open Hermite snakes with simple broken lines, we were able to accurately detect the medial axis, as shown in Figure 9. Snake optimization was carried out relying on (35) for cubic spline snakes, and on the proposed directional energy functional (28) for Hermite snakes, using in both cases properly tuned ridge-sensitive steerable filters. As they do not incorporate any directional information, the cubic spline snakes are more likely to get trapped in local minima and yield a less good estimation of the medial axis.

2) Segmentation of Phase-Contrast Microscopic Images: We segmented phase-contrast microscopic images of HeLa cells from [36]. Phase-contrast images are challenging for segmentation as they feature uneven gradients and halos around objects. We initialized closed Hermite snakes with rough polygonal shapes around the cells, setting the number of control points as the number of edges in the polygon. Tangents at each points were initially set to zero. We then optimized the exponential snakes on (35) and the Hermite snakes on the directional energy functional (28), both using an edge-sensitive steerable filter for the magnitude information. Results are displayed in Figure 10. Note that orientation information is of particular importance in these images as cell shapes exhibit fine details, in analogy with the synthetic example of Figure 7.

3) Segmentation of Differential-Interference-Contrast Images: Similar to phase-contrast microscopy, differential interference contrast (DIC) microscopy yields images where object boundaries are uneven due to shading effects.

\footnotetext{
${ }^{4}$ Images courtesy of J. Esser, Laboratory of Intestinal Immunology, École polytechnique fédérale de Lausanne (EPFL), Switzerland.
} 
TABLE I

JacCard Index for Noisy Data In the Closed- And Open-Snake Cases, as a Function of $M$

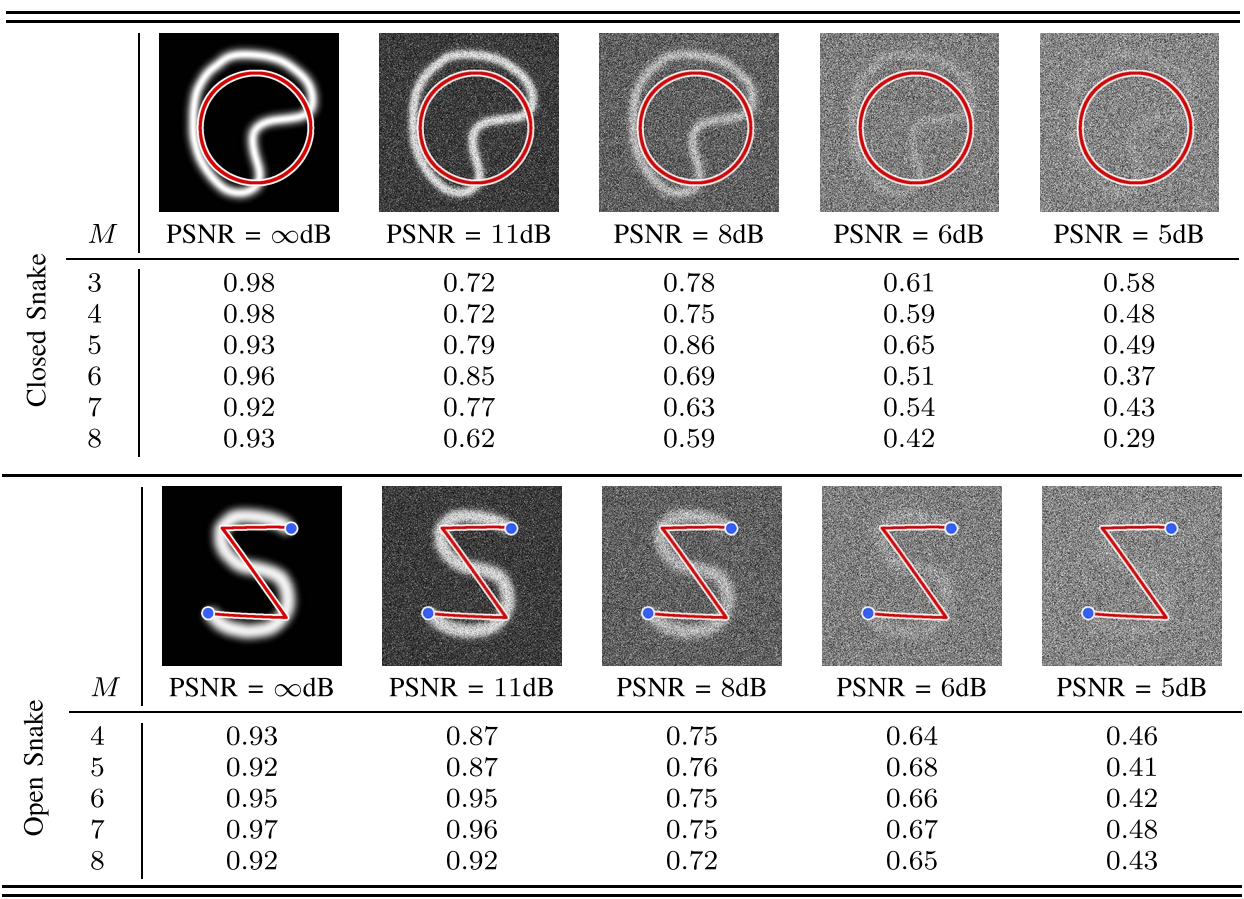
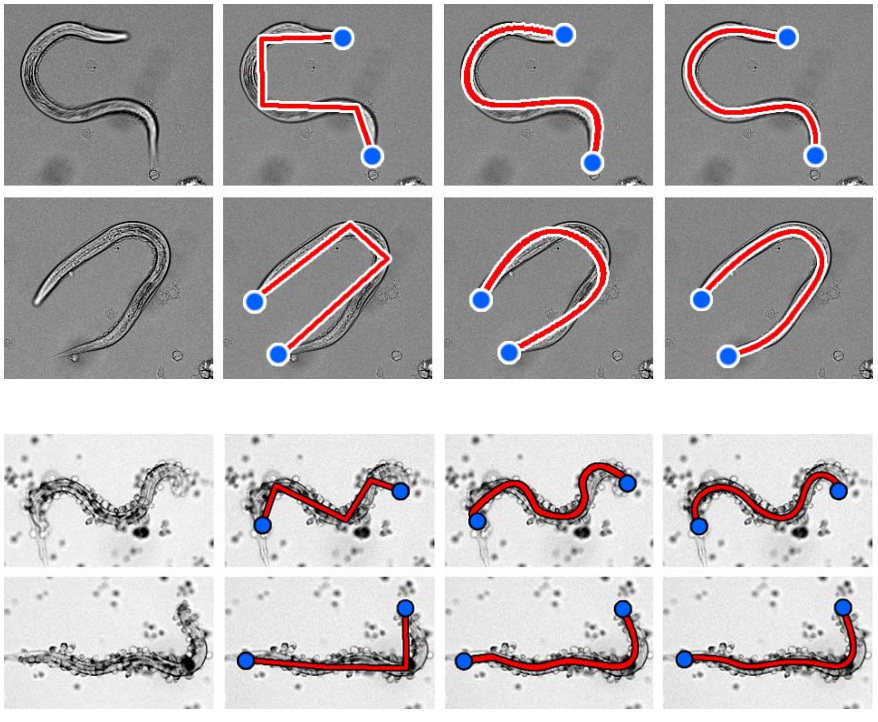

Fig. 9. Outline of $H$. bakeri medial axis using open Hermite snakes in clean (top rows) and cluttered cases (bottom rows). From left to right: original image, snake initialization, automated segmentation using a classical cubic B-splines snake, automated segmentation using the Hermite snake. Frozen points are depicted as disks.

We obtained images of pancreatic acinar cells of live guinea pigs from the Cell Image Library ${ }^{5}$ and initialized circular closed Hermite snakes inside each cell. Each snake contained 5 control points and their associated tangents for Hermite snakes. Relying on (35) for exponential snakes and on our directional energy functional (28), both using an edge-sensitive steerable filter, we obtained the segmentation results shown in Figure 11. Here again, the possibility to

\footnotetext{
${ }^{5}$ Image corresponding to [37, Fig. 1], freely accessible from the Cell Image Library (http://www.cellimagelibrary.org/), accession number CIL:37314.
}
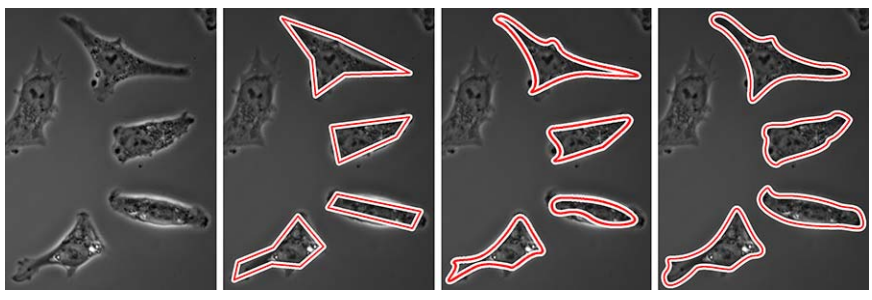

Fig. 10. Segmentation of phase-contrast images of HeLa cells using closed Hermite snakes. From left to right: original image, snake initialization, automated segmentation result using a classical spline-snake [14], automated segmentation result using the Hermite snake.

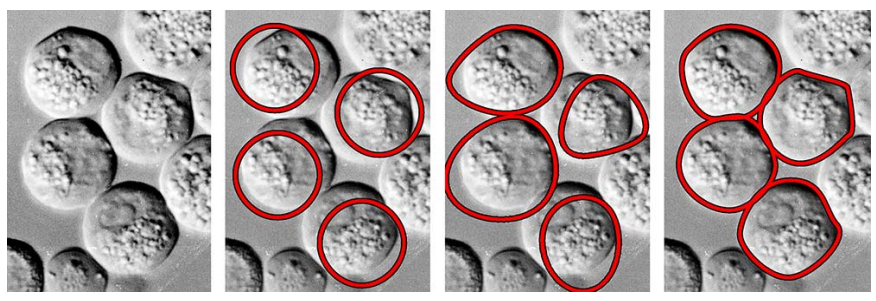

Fig. 11. Segmentation of differential-interference-contrast images of animal pancreatic acinar cells using closed Hermite snakes. From left to right: original image, snake initialization, automated segmentation result using a classical spline-snake [14], automated segmentation result using the Hermite snake.

add orientation information is crucial to constrain the overall shape of the snake in areas where cells touch each other and where little gradient information is available, similarly to the situation in Figure 8.

4) Outline of Polygonal Cells: Finally, we analyzed scanning-electron micrograph of the epidermal surface of lamprey larvae. ${ }^{6}$ The image features microvilli that outline the polygonal borders between cells, while short microvilli cover

${ }^{6}$ Image from [38], freely accessible from the Cell Image Library, accession number CIL:11115. 


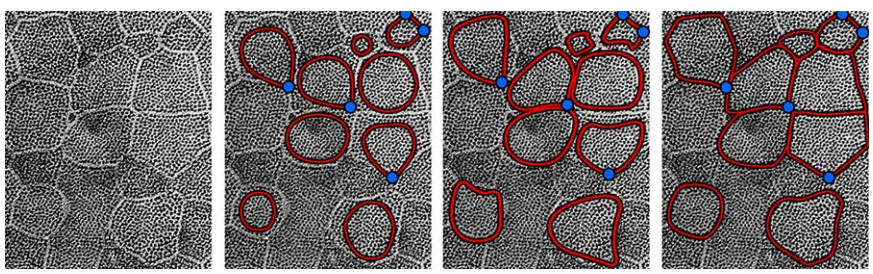

Fig. 12. Outline of borders of epidermal cells using closed Hermite snakes. From left to right: original image, snake initialization, automated segmentation result using a classical spline-snake [14], automated segmentation result using the Hermite snake. Frozen points are depicted as disks.

the external surface in a reticular network. The motivation for using Hermite snakes in these data is the presence of polygonal cells with sharp corners. We therefore initialized closed Hermite snakes inside the cells and manually imposed some critical corners. The number of control points was tuned depending on the complexity of each cell. We similarly initialized exponential snakes and imposed the same fixed points. The exponential snake curves were composed of at most twice more control points than the corresponding Hermite snakes in order to allow for the same curve flexibility. The snakes were then automatically optimized. As shown in Figure 12, the Hermite snake is able to properly segment both smooth and polygonal cell. It can hence better accommodate biological variability than active contours that are uniformly smooth by construction and therefore require many control points to generate discontinuities, which in turn increases the risk of entanglement.

\section{Conclusions}

We introduced in this paper a new snake that relies on interpolating Hermite splines. Our model is able to handle open and closed 2-D curves that can be used for the semiautomated segmentation of bioimages. The novel feature of our Hermite snake is the explicit introduction of tangent controls in the contour. The advantages are twofold. Firstly, it allows for sharp corners in the active contour. Secondly, it enables the design of directional energy functionals to better guide the snake during automated segmentation. On the theoretical side, we have provided a formal exploration of the Hermite snake construction. We motivate its practical usefulness and highlight its connections with existing contour representations. On the practical side, we have compared our model against existing ones and studied its dependency on various parameters. Finally, we have showed its practical usefulness in different bioimage data where the use of the Hermite snake is of particular interest. There, we observed that Hermite snakes perform better than their classical counterparts when the intensity information is ambiguous or not sufficiently uniform, or when objects exhibits sharp corners. This can be attributed to the joint use of the proposed snake model and directional energy functional.

We conclude with some remarks about the limitations of our model and possible future work. The tangent vectors of the Hermite snake grant more flexibility to the curve and enable the generation of corners. However, they also results in a large number of parameters. The Hermite snake must thus be properly constrained during optimization. The proposed directional energy functional fulfills this task, but existing energies for classical spline snakes that do not include tangents are likely to be insufficient for preventing the snake from diverging. Future work around the Hermite snake would therefore include deriving more energy functionals involving tangents and, more generally, proposing internal energies regularizing the behavior of the tangent field of our model.

\section{APPENDIX A}

\section{Link With Classical Splines}

Relying on the well-known link between the Green's function of a given operator and its associated spline [39], we aim here at stating the relation between the Hermite spline generators $\boldsymbol{\phi}=\left(\phi_{1}, \phi_{2}\right)$ and $\rho=\left(\rho_{\mathrm{D}^{4}}, \rho_{\mathrm{D}^{3}}\right)$, which are the Green's functions of the operators that are associated to the classical cubic and quadratic splines $\left(\mathrm{D}^{4}=\frac{\mathrm{d}^{4}}{\mathrm{~d} t^{4}}\right.$ and $\mathrm{D}^{3}=\frac{\mathrm{d}^{3}}{\mathrm{~d} t^{3}}$, respectively $)$. The explicit expressions of their Green's functions are

$$
\begin{aligned}
& \rho_{\mathrm{D}^{4}}(t)=\mathcal{F}^{-1}\left\{\frac{1}{(\mathrm{j} \cdot)^{4}}\right\}(t)=\frac{1}{12} t^{3} \operatorname{sgn}(t), \\
& \rho_{\mathrm{D}^{3}}(t)=\mathcal{F}^{-1}\left\{\frac{1}{(\mathrm{j} \cdot)^{3}}\right\}(t)=\frac{1}{4} t^{2} \operatorname{sgn}(t) .
\end{aligned}
$$

By inverting the $(2 \times 2)$ Fourier matrix $\widehat{\mathbf{R}}\left(\mathrm{e}^{\mathrm{j} \omega}\right)$ from (15), we find that

$$
\widehat{\boldsymbol{\rho}}(\omega)=\widehat{\mathbf{R}}\left(\mathrm{e}^{\mathrm{j} \omega}\right)^{-1} \widehat{\boldsymbol{\phi}}(\omega) .
$$

Since $\widehat{\mathbf{R}}\left(\mathrm{e}^{\mathrm{j} \omega}\right)^{-1}=\widehat{\mathbf{S}}\left(\mathrm{e}^{\mathrm{j} \omega}\right)$ has entries that are ratios of trigonometric polynomials, its discrete-time inverse Fourier transform is well-defined and guaranteed to yield a unique sequence of matrices $\mathbf{S}[k]=\frac{1}{2 \pi} \int_{-\pi}^{+\pi} \widehat{\mathbf{S}}\left(\mathrm{e}^{\mathrm{j} \omega}\right) \mathrm{e}^{\mathrm{j} \omega k} \mathrm{~d} \omega$ of slow growth. Hence, we conclude that

$$
\boldsymbol{\rho}(t)=\sum_{k \in \mathbb{Z}} \mathbf{S}[k] \boldsymbol{\phi}(t-k),
$$

which proves that the Green's functions $\rho_{\mathrm{D}^{4}}$ and $\rho_{\mathrm{D}^{3}}$, as well as their integer shifts, can be perfectly reproduced by $\{\phi(\cdot-k)\}_{k \in \mathbb{Z}}$. The specific form of (39) then follows from the interpolation property of the generators; that is, the relation

$$
s(t)=\sum_{k \in \mathbb{Z}}\left(s(k) \phi_{1}(t-k)+s^{\prime}(k) \phi_{2}(t-k)\right),
$$

which is valid for any function in the span of the cubic Hermite splines. In particular, we have that

$$
\begin{aligned}
\rho_{\mathrm{D}^{4}}(t)= & \frac{1}{12} t^{3} \operatorname{sgn}(t) \\
=\sum_{k \in \mathbb{Z}}\left(\frac{1}{12} k^{3} \operatorname{sgn}(k) \phi_{1}(t-k)\right. & \\
& \left.\quad+\frac{1}{4} k^{2} \operatorname{sgn}(k) \phi_{2}(t-k)\right) .
\end{aligned}
$$

It is known [26] that a cardinal cubic spline admits a unique stable expansion of the type

$$
s(t)=\sum_{k \in \mathbb{Z}} a[k] \rho_{\mathrm{D}^{4}}(t-k),
$$


where $a$ is a sequence of coefficients of slow growth. This hence implies that the space of cubic Hermite splines includes Schoenberg's space of cubic splines as a subspace. The same holds true for the quadratic splines which are generated by $\left\{\rho_{\mathrm{D}^{3}}(\cdot-k)\right\}_{k \in \mathbb{Z}}$.

Based on (13), we can express $\phi_{1}$ as

$$
\phi_{1}=12 \Delta_{+}^{2} \rho_{\mathrm{D}^{4}}+6 \Delta_{+} \rho_{\mathrm{D}^{3}} .
$$

The expression of $\phi_{2}$ in terms of $\rho_{\mathrm{D}^{4}}$ and $\rho_{\mathrm{D}^{3}}$ can be obtained through an analogous calculation. We conclude that the Hermite spline space can be seen as a space of cubic splines that can accommodate quadratic spline-like transitions.

The causal cubic B-spline can then be written in the two following equivalent ways

$$
\begin{aligned}
\beta_{+}^{3}(t)= & \Delta_{+}^{4} \rho_{\mathrm{D}^{4}}(t) \\
= & \frac{1}{6} \phi_{1}(t-1)+\frac{4}{6} \phi_{1}(t-2)+\frac{1}{6} \phi_{1}(t-3) \\
& +\frac{1}{2} \phi_{2}(t-1)-\frac{1}{2} \phi_{2}(t-2),
\end{aligned}
$$

where $\Delta_{+}^{4}$ is the 4 th-order finite-difference operator with $\Delta_{+} f(t)=f(t)-f(t-1)$. Since the cubic B-splines reproduce the polynomials of degree 3 , the property automatically extends to the Hermite spline space, which yields

$$
t^{m}=\sum_{k \in \mathbb{Z}}\left(k^{m} \phi_{1}(t-k)+m k^{m-1} \phi_{2}(t-k)\right)
$$

for $m=0,1,2,3$. Similarly, we can make use of the Hermite interpolation property (40) to obtain the corresponding expression for the quadratic B-spline,

$$
\begin{aligned}
\beta_{+}^{2}(t) & =\Delta_{+}^{3} \rho_{\mathrm{D}^{3}}(t) \\
& =\frac{1}{2} \phi_{1}(t-1)+\frac{1}{2} \phi_{1}(t-2)+\phi_{2}(t-1)-\phi_{2}(t-2),
\end{aligned}
$$

which is supported in $[0,3]$.

The above expressions help us understand another remarkable property of the Hermite spline space with respect to the theory of conventional splines. In the case of the cubic splines, a compactly supported basis function (i.e., a B-spline) is constructed by applying the discrete version $\Delta_{+}^{4}$ of the operator $\mathrm{D}^{4}$ to its Green's function $\rho_{\mathrm{D}^{4}}$, as described by (44). Quadratic B-splines are constructed in the same way by applying the discrete version $\Delta_{+}^{3}$ of the operator $\mathrm{D}^{3}$ to the Green's function $\rho_{\mathrm{D}^{3}}$. While either of the functions $\Delta_{+}^{2} \rho_{\mathrm{D}^{4}}$ and $\Delta_{+} \rho_{\mathrm{D}^{3}}$ is only partially localized and still includes a linear trend, the combination of both results in the cancellation of all residual polynomial components. Consequently, the Hermite basis functions have a support of size 2, which is shorter than the classical B-splines.

\section{APPENDIX B}

\section{RIESZ-BASIS PROPERTY}

The Riesz basis property is best verified in the Fourier domain. To that end, we first compute the Fourier Gram matrix of the basis, which is given by

$$
\widehat{\mathbf{G}}\left(\mathrm{e}^{\mathrm{j} \omega}\right)
$$

$$
\begin{aligned}
& =\sum_{k \in \mathbb{Z}} \widehat{\boldsymbol{\phi}}(\omega+2 \pi k) \widehat{\boldsymbol{\phi}}(\omega+2 \pi k)^{H} \\
& =\left[\begin{array}{cc}
\sum_{k \in \mathbb{Z}}\left\langle\phi_{1}, \phi_{1}(\cdot-k)\right\rangle \mathrm{e}^{-\mathrm{j} \omega k} & \sum_{k \in \mathbb{Z}}\left\langle\phi_{1}, \phi_{2}(\cdot-k)\right\rangle \mathrm{e}^{-\mathrm{j} \omega k} \\
\sum_{k \in \mathbb{Z}}\left\langle\phi_{2}, \phi_{1}(\cdot-k)\right\rangle \mathrm{e}^{-\mathrm{j} \omega k} & \sum_{k \in \mathbb{Z}}\left\langle\phi_{2}, \phi_{2}(\cdot-k)\right\rangle \mathrm{e}^{-\mathrm{j} \omega k}
\end{array}\right] \\
& =\left[\begin{array}{cc}
\frac{26}{35}+\frac{9 \mathrm{e}^{-\mathrm{j} \omega}}{70}+\frac{9 \mathrm{e}^{\mathrm{j} \omega}}{70} & -\frac{13 \mathrm{e}^{-\mathrm{j} \omega}}{420}+\frac{13 \mathrm{e}^{\mathrm{j} \omega}}{420} \\
\frac{13 \mathrm{e}^{-\mathrm{j} \omega}}{420}-\frac{13 \mathrm{e}^{\mathrm{j} \omega}}{420} & \frac{2}{105}-\frac{\mathrm{e}^{-i \omega}}{140}-\frac{\mathrm{e}^{\mathrm{j} \omega}}{140}
\end{array}\right] .
\end{aligned}
$$

The Gram matrix $\widehat{\mathbf{G}}\left(\mathrm{e}^{\mathrm{j} \omega}\right)$ is Hermitian symmetric and $2 \pi$-periodic in $\omega$. Next, we recall that the Fourier equivalent of the Riesz-basis requirement (16) is

$0<A^{2}=\min _{\omega \in[0, \pi]} \lambda_{\min }\left(\mathrm{e}^{\mathrm{j} \omega}\right) \leq \max _{\omega \in[0, \pi]} \lambda_{\max }\left(\mathrm{e}^{\mathrm{j} \omega}\right)=B^{2}<+\infty$,

where $\lambda_{\max }\left(\mathrm{e}^{\mathrm{j} \omega}\right)$ and $\lambda_{\min }\left(\mathrm{e}^{\mathrm{j} \omega}\right)$ denote the maximum and minimum eigenvalues of $\widehat{\mathbf{G}}\left(\mathrm{e}^{\mathrm{j} \omega}\right)$ at frequency $\omega$, respectively. Moreover, the constants $A$ and $B$ in (48) are the optimal ones. This result is classical for the case of a single generator $\varphi$; see for instance [40], [41]. In that case, we have $\lambda_{\min }\left(\mathrm{e}^{\mathrm{j} \omega}\right)=\lambda_{\max }\left(\mathrm{e}^{\mathrm{j} \omega}\right)=\sum_{k \in \mathbb{Z}}|\widehat{\varphi}(\omega+2 \pi k)|^{2}$. The case of several generators, which is required in the Hermite scenario, is covered by [42, Th. 2.1]. Knowing the Gram matrix (47), we simply compute the minimum and maximum eigenvalues and take the supremum and infimum among $\omega \in[0, \pi]$. The calculation yields the exact value of the Riesz bounds, namely

$$
(A, B)=\left(210^{-\frac{1}{2}}, 1\right)
$$

with the worst case of (48) being found at $\omega=0$.

\section{APPENDIX C}

\section{INHERENT SMOOTHNESS PROPERTY}

We here give the proof of Theorem 1. First, we have to ensure that the Hermite interpolant $s(\cdot)$ exists for fixed $s[\cdot], s^{\prime}[\cdot]$ and is uniquely defined. This is already ensured by Schoenberg's work dedicated to the cubic Hermite splines [23], [43]. Then, we have to show that, for any $f$ satisfying the two interpolation constraints $f(k)=s[k]$ and $f^{\prime}(k)=s^{\prime}[k]$, we have

$$
\left\|\mathrm{D}^{2} f\right\|_{L_{2}}^{2}=\left\|\mathrm{D}^{2} s\right\|_{L_{2}}^{2}+\left\|\mathrm{D}^{2}(f-s)\right\|_{L_{2}}^{2},
$$

where $\mathrm{D}^{2}$ denotes the second derivative operator $\frac{\mathrm{d}^{2}}{\mathrm{~d} t^{2}}$. Hence, if $f=s$, the second term in (50) vanishes, implying that $s$ is optimal among all solutions that interpolate $f$. Let us take $g=f-s$. Then,

$$
\left\|\mathrm{D}^{2} g+\mathrm{D}^{2} s\right\|_{L_{2}}^{2}=\left\|\mathrm{D}^{2} s\right\|_{L_{2}}^{2}+2\left\langle\mathrm{D}^{2} s, \mathrm{D}^{2} g\right\rangle_{L_{2}}+\left\|\mathrm{D}^{2} g\right\|_{L_{2}}^{2},
$$

and proving (50) reduces to demonstrating that

$$
\left\langle\mathrm{D}^{2} s, \mathrm{D}^{2} g\right\rangle_{L_{2}}=0 .
$$

By definition, $s$ is given by

$$
s(t)=\sum_{k \in \mathbb{Z}}\left(s[k] \phi_{1}(t-k)+s^{\prime}[k] \phi_{2}(t-k)\right)
$$




$$
\begin{aligned}
= & \left(\phi_{1} * \sum_{k \in \mathbb{Z}} s[k] \delta(\cdot-k)\right)(t) \\
& +\left(\phi_{2} * \sum_{k \in \mathbb{Z}} s^{\prime}[k] \delta(\cdot-k)\right)(t),
\end{aligned}
$$

where $*$ denotes the continuous convolution and $\delta$ is Dirac's delta. In the Fourier domain,

$$
\begin{aligned}
\widehat{s}(\omega) & =\widehat{\phi}_{1}(\omega) S_{1}\left(\mathrm{e}^{\mathrm{j} \omega}\right)+\widehat{\phi}_{2}(\omega) S_{2}\left(\mathrm{e}^{\mathrm{j} \omega}\right) \\
& =\widehat{\boldsymbol{\phi}}^{T}(\omega)\left[\begin{array}{l}
S_{1}\left(\mathrm{e}^{\mathrm{j} \omega}\right) \\
S_{2}\left(\mathrm{e}^{\mathrm{j} \omega}\right)
\end{array}\right],
\end{aligned}
$$

with $S_{1}\left(\mathrm{e}^{\mathrm{j} \omega}\right), S_{2}\left(\mathrm{e}^{\mathrm{j} \omega}\right)$ the discrete Fourier transforms of the sequences $s$ and $s^{\prime}$, respectively. Since, from (38)

$$
\widehat{\boldsymbol{\phi}}(\omega)=\widehat{\mathbf{R}}\left(\mathrm{e}^{\mathrm{j} \omega}\right) \widehat{\boldsymbol{\rho}}(\omega)=\widehat{\mathbf{R}}\left(\mathrm{e}^{\mathrm{j} \omega}\right)\left[\begin{array}{c}
1 \\
\mathrm{j} \omega
\end{array}\right] \widehat{\rho}_{\mathrm{D}^{4}}(\omega),
$$

then

$$
\widehat{s}(\omega)=\left[\begin{array}{c}
1 \\
\mathrm{j} \omega
\end{array}\right]^{T} \widehat{\rho}_{\mathrm{D}^{4}}(\omega) \widehat{\mathbf{R}}^{T}\left(\mathrm{e}^{\mathrm{j} \omega}\right)\left[\begin{array}{l}
S_{1}\left(\mathrm{e}^{\mathrm{j} \omega}\right) \\
S_{2}\left(\mathrm{e}^{\mathrm{j} \omega}\right)
\end{array}\right] .
$$

In addition, we note that $\left\langle\mathrm{D}^{2} s, \mathrm{D}^{2} g\right\rangle_{L_{2}}=\left\langle s,\left(\mathrm{D}^{2}\right)^{*} \mathrm{D}^{2} g\right\rangle_{L_{2}}=$ $\left\langle s, \mathrm{D}^{4} g\right\rangle_{L_{2}}$. Also, let us remind that $\mathrm{j} \omega \widehat{g}(\omega)=\widehat{g^{\prime}}(\omega)$. Then, relying on Parseval's theorem and on the Cauchy-Schwarz inequality,

$$
\begin{aligned}
\left\langle s, \mathrm{D}^{4} g\right\rangle_{L_{2}} & \frac{1}{2 \pi} \int_{\mathbb{R}} \widehat{s}(\omega)(\mathrm{j} \omega)^{4} \widehat{g}(\omega) \mathrm{d} \omega \\
= & \frac{1}{2 \pi} \int_{\mathbb{R}}\left[\begin{array}{c}
1 \\
\mathrm{j} \omega
\end{array}\right]^{T} \widehat{\mathbf{R}}^{T}\left(\mathrm{e}^{\mathrm{j} \omega}\right)\left[\begin{array}{c}
S_{1}\left(\mathrm{e}^{\mathrm{j} \omega}\right) \\
S_{2}\left(\mathrm{e}^{\mathrm{j} \omega}\right)
\end{array}\right] \widehat{g}(\omega) \mathrm{d} \omega \\
= & \frac{1}{2 \pi} \int_{\mathbb{R}}\left\langle\widehat{\mathbf{R}}\left(\mathrm{e}^{\mathrm{j} \omega}\right)\left[\begin{array}{c}
S_{1}\left(\mathrm{e}^{\mathrm{j} \omega}\right) \\
S_{2}\left(\mathrm{e}^{\mathrm{j} \omega}\right)
\end{array}\right],\left[\begin{array}{c}
\widehat{g}(\omega) \\
\widehat{g^{\prime}}(\omega)
\end{array}\right]\right\rangle_{L_{2}} \mathrm{~d} \omega \\
= & \frac{1}{2 \pi} \int_{0}^{2 \pi} A\left(\mathrm{e}^{\mathrm{j} \omega}\right) \sum_{k \in \mathbb{Z}} \widehat{g}(\omega+2 k \pi) \mathrm{d} \omega \\
& +\frac{1}{2 \pi} \int_{0}^{2 \pi} B\left(\mathrm{e}^{\mathrm{j} \omega}\right) \sum_{k \in \mathbb{Z}} \widehat{g^{\prime}}(\omega+2 k \pi) \mathrm{d} \omega \\
\leq & \left\|A\left(\mathrm{e}^{\mathrm{j} \omega}\right)\right\|_{L_{2}([0,2 \pi])}\left\|\sum_{k \in \mathbb{Z}} \widehat{g}(\omega+2 k \pi)\right\| \|_{L_{2}([0,2 \pi])}, \\
& \times\left\|B\left(\mathrm{e}^{\mathrm{j} \omega}\right)\right\|_{L_{2}([0,2 \pi])}\left\|\sum_{k \in \mathbb{Z}} \widehat{g^{\prime}}(\omega+2 k \pi)\right\|_{L_{2}([0,2 \pi])}
\end{aligned}
$$

where $A=\widehat{R}_{11} S_{1}+\widehat{R}_{12} S_{2}$ and $B=\widehat{R}_{21} S_{1}+\widehat{R}_{22} S_{2}$. The terms $\|A\|_{L_{2}([0,2 \pi])}$ and $\|B\|_{L_{2}([0,2 \pi])}$ are bounded as the four entries of $\widehat{\mathbf{R}}$ given by (38) have finite $L_{2}$ norms, and the $S_{1}\left(\mathrm{e}^{\mathrm{j} \omega}\right)$, $S_{2}\left(\mathrm{e}^{\mathrm{j} \omega}\right)$ are discrete-time Fourier transforms of sequences $s, s^{\prime} \in \ell_{2}(\mathbb{Z})$. Finally, since $g=f-s$ and $f(n)=s(n)$, $f^{\prime}(n)=s^{\prime}(n)$ for all $n \in \mathbb{Z}$, one has by Poisson formula

$$
\begin{aligned}
& \sum_{k \in \mathbb{Z}} \widehat{g}(\omega+2 k \pi)=\sum_{k \in \mathbb{Z}} g(n) \mathrm{e}^{-\mathrm{j} n \omega}=0, \\
& \sum_{k \in \mathbb{Z}} \widehat{g^{\prime}}(\omega+2 k \pi)=\sum_{k \in \mathbb{Z}} g^{\prime}(n) \mathrm{e}^{-\mathrm{j} n \omega}=0 .
\end{aligned}
$$

This concludes the proof of Theorem 1 .

\section{ACKNOWLEDGMENT}

The authors would like to thank members of the Biomedical Imaging Group R. Delgado-Gonzalo and D. Schmitter for fruitful discussions about the structure of the manuscript.

\section{REFERENCES}

[1] M. Kass, A. Witkin, and D. Terzopoulos, "Snakes: Active contour models," Int. J. Comput. Vis., vol. 1, no. 4, pp. 321-331, Jan. 1988.

[2] R. Delgado-Gonzalo, V. Uhlmann, D. Schmitter, and M. Unser, "Snakes on a plane: A perfect snap for bioimage analysis," IEEE Signal Process. Mag., vol. 32, no. 1, pp. 41-48, Jan. 2015.

[3] A. K. Jain, Y. Zhong, and M.-P. Dubuisson-Jolly, "Deformable template models: A review," Signal Process., vol. 71, no. 2, pp. 109-129, Dec. 1998.

[4] S. Menet, P. Saint-Marc, and G. Medioni, "Active contour models: Overview, implementation and applications," in Proc. IEEE Int. Conf. Syst., Man Cybern. (SMC), Los Angeles, CA, USA, Nov. 1990, pp. 194-199.

[5] N. Ray, B. Chanda, and J. Das, "A fast and flexible multiresolution snake with a definite termination criterion," Pattern Recognit., vol. 34, no. 7, pp. 1483-1490, 2001.

[6] L. D. Cohen and R. Kimmel, "Global minimum for active contour models: A minimal path approach," Int. J. Comput. Vis., vol. 24, no. 1, pp. 57-78, Aug. 1997.

[7] M. A. T. Figueiredo, J. M. N. Leito, and A. K. Jain, "Unsupervised contour representation and estimation using B-splines and a minimum description length criterion," IEEE Trans. Image Process., vol. 9, no. 6, pp. 1075-1087, Jun. 2000.

[8] B. Li and S. T. Acton, "Active contour external force using vector field convolution for image segmentation," IEEE Trans. Image Process., vol. 16, no. 8, pp. 2096-2106, Aug. 2007.

[9] N. Ray and S. T. Acton, "Motion gradient vector flow: An external force for tracking rolling leukocytes with shape and size constrained active contours," IEEE Trans. Med. Imag., vol. 23, no. 12, pp. 1466-1478, Dec. 2004.

[10] W. Dahmen, B. Han, R.-Q. Jia, and A. Kunoth, "Biorthogonal multiwavelets on the interval: Cubic hermite splines," Constructive Approx. vol. 16, no. 2, pp. 221-259, Feb. 2000.

[11] V. Uhlmann, R. Delgado-Gonzalo, and M. Unser, "Snakes with tangentbased control and energies for bioimage analysis," in Proc. 11th IEEE Int. Symp. Biomed. Imagi., Nano Macro (ISBI), Beijing, China, Apr./May 2014, pp. 806-809.

[12] P. Brigger, J. Hoeg, and M. Unser, "B-spline snakes: A flexible tool for parametric contour detection," IEEE Trans. Signal Process., vol. 9, no. 9, pp. 1484-1496, Sep. 2000

[13] M. Jacob, T. Blu, and M. Unser, "Efficient energies and algorithms for parametric snakes," IEEE Trans. Image Process., vol. 13, no. 9, pp. 1231-1244, Sep. 2004.

[14] R. Delgado-Gonzalo, P. Thévenaz, C. S. Seelamantula, and M. Unser, "Snakes with an ellipse-reproducing property," IEEE Trans. Image Process., vol. 21, no. 3, pp. 1258-1271, Mar. 2012.

[15] R. Kimmel and A. M. Bruckstein, "Regularized Laplacian zero crossings as optimal edge integrators," Int. J. Comput. Vis., vol. 53, no. 3, pp. 225-243, Jul. 2003.

[16] M. D. Abràmoff, P. J. Magalhães, and S. J. Ram, "Image processing with ImageJ," Biophoton. Int., vol. 11, no. 7, pp. 36-42, Jul. 2004.

[17] M. Unser, "Sampling-50 years after Shannon," Proc. IEEE, vol. 88, no. 4, pp. 569-587, Apr. 2000.

[18] R. Delgado-Gonzalo and M. Unser, "Spline-based framework for interactive segmentation in biomedical imaging," IRBM-Ingénierie Recherche Biomédicale/BioMed. Eng. Res., vol. 34, no. 3, pp. 235-243, Jun. 2013.

[19] W. M. Neuenschwander, P. Fua, L. Iverson, and G. Székely, and O. Kübler, "Ziplock snakes," Int. J. Comput. Vis., vol. 25, no. 3, pp. 191-201, Dec. 1997.

[20] Y. Y. Wong, P. C. Yuen, and C. S. Tong, "Segmented snake for contour detection," Pattern Recognit., vol. 31, no. 11, pp. 1669-1679, Nov. 1998.

[21] M. B. Smith, H. Li, T. Shen, X. Huang, E. Yusuf, and D. Vavylonis, "Segmentation and tracking of cytoskeletal filaments using open active contours," Cytoskeleton, vol. 67, no. 11, pp. 693-705, Nov. 2010.

[22] J. Melonakos, E. Pichon, S. Angenent, and A. Tannenbaum, "Finsler active contours," IEEE Trans. Pattern Anal. Mach. Intell., vol. 30, no. 3, pp. 412-423, Mar. 2008. 
[23] P. R. Lipow and I. J. Schoenberg, "Cardinal interpolation and spline functions. III. Cardinal Hermite interpolation," Linear Algebra Appl., vol. 6, pp. 273-304, 1973. [Online]. Available: http://www.sciencedirect.com/science/journal/00243795/6/supp/C

[24] V. Uhlmann, R. Delgado-Gonzalo, C. Conti, L. Romani, and M. Unser, "Exponential Hermite splines for the analysis of biomedical images," in Proc. 39th IEEE Int. Conf. Acoust., Speech, Signal Process. (ICASSP), Florence, Italy, May 2014, pp. 1631-1634.

[25] C. Conti, L. Romani, and M. Unser, "Ellipse-preserving Hermite interpolation and subdivision," J. Math. Anal. Appl., vol. 426, no. 1, pp. 211-227, Jun. 2015.

[26] M. Unser and P. D. Tafti, "Splines and wavelets," in An Introduction to Sparse Stochastic Processes. Cambridge, U.K.: Cambridge Univ. Press, Oct. 2014, ch. 6, pp. 112-148.

[27] T. Blu and M. Unser, "Approximation error for quasi-interpolators and (multi-)wavelet expansions," Appl. Comput. Harmon. Anal., vol. 6, no. 2, pp. 219-251, Mar. 1999.

[28] A. Chakraborty, L. H. Staib, and J. S. Duncan, "Deformable boundary finding in medical images by integrating gradient and region information," IEEE Trans. Med. Imag., vol. 15, no. 6, pp. 859-870, Dec. 1996.

[29] L. H. Staib and J. S. Duncan, "Boundary finding with parametrically deformable models," IEEE Trans. Pattern Anal. Mach. Intell., vol. 14, no. 11, pp. 1061-1075, Nov. 1992.

[30] J. Gao, A. Kosaka, and A. Kak, "A deformable model for human organ extraction," in Proc. IEEE Int. Conf. Image Process. (ICIP), vol. 1. Chicago, IL, USA, Oct. 1998, pp. 323-327.

[31] J. Ahlberg, E. Nilson, and J. Walsh, "Intrinsic properties of cubic splines," in The Theory of Splines and Their Applications (Mathematics in Science and Engineering), vol. 38. New York, NY, USA: Academic, 1967, pp. 75-108.

[32] G. B. Arfken, Mathematical Methods for Physicists, 3rd ed. Orlando, FL, USA: Academic, 1985.

[33] R. Ronfard, "Region-based strategies for active contour models," Int. J. Comput. Vis., vol. 13, no. 2, pp. 229-251, Oct. 1994.

[34] M. Jacob and M. Unser, "Design of steerable filters for feature detection using canny-like criteria," IEEE Trans. Pattern Anal. Mach. Intell., vol. 26, no. 8, pp. 1007-1019, Aug. 2004.

[35] W. H. Press, S. A. Teukolsky, W. T. Vetterling, and B. P. Flannery, Numerical Recipes: The Art of Scientific Computing, 3rd ed. Cambridge, U.K.: Cambridge Univ. Press, 1986, p. 818.

[36] E. Bostan, E. Froustey, B. Rappaz, E. Shaffer, D. Sage, and M. Unser, "Phase retrieval by using transport-of-intensity equation and differential interference contrast microscopy," in Proc. IEEE Int. Conf. Image Process. (ICIP), Paris, France, Oct. 2014, pp. 3939-3943.

[37] A. Amsterdam and J. D. Jamieson, "Structural and functional characterization of isolated pancreatic exocrine cells," Proc. Nat. Acad. Sci. USA, vol. 69, no. 10, pp. 3028-3032, Oct. 1972.

[38] W. H. Fahrenbach and D. D. Knutson, "Surface adaptations of the vertebrate epidermis to friction," J. Investigative Dermatol., vol. 65 , no. 1, pp. 39-44, Jun. 1975.

[39] M. Unser, "Splines: A perfect fit for signal and image processing," IEEE Signal Process. Mag., vol. 16, no. 6, pp. 22-38, Nov. 1999.

[40] A. Aldroubi, M. Unser, and A. Aldroubi, "Sampling procedures in function spaces and asymptotic equivalence with Shannon's sampling theory," Numer. Funct. Anal. Optim., vol. 15, nos. 1-2, pp. 1-21, 1994.

[41] A. Aldroubi and K. Gröchenig, "Nonuniform sampling and reconstruction in shift-invariant spaces," SIAM Rev., vol. 43, no. 4, pp. 585-620, Dec. 2001

[42] A. Aldroubi, "Oblique projections in atomic spaces," Proc. Amer. Math. Soc., vol. 124, no. 7, pp. 2051-2060, Jul. 1996.

[43] I. J. Schoenberg and A. Sharma, "Cardinal interpolation and spline functions V. The B-splines for cardinal Hermite interpolation," Linear Algebra Appl., vol. 7, no. 1, pp. 1-42, Jan. 1973.

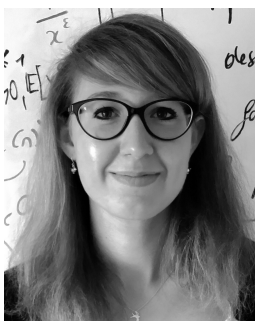

Virginie Uhlmann received the M.Sc. degree in bioengineering from the École Polytechnique Fédérale de Lausanne (EPFL), Switzerland, in 2012, where she is currently pursuing the Ph.D. degree with the Biomedical Imaging Group under the direction of M. Unser. She completed her master's thesis in the Imaging Platform with the Broad Institute, Cambridge, MA, under the supervision of A. Carpenter. She is working on applied problem related to image segmentation and tracking, and on approximation and spline theory. Her research interests include image processing, computer vision, machine learning, and life sciences.

She received the competitive Excellence Fellowship at the Master's level from EPFL in 2011 and 2012, and the Best Student Paper Award from the 2014 IEEE International Conference on Image Processing. She also received a Best Student Paper Award Nomination from the IEEE International Symposium on Biomedical Imaging in 2015.

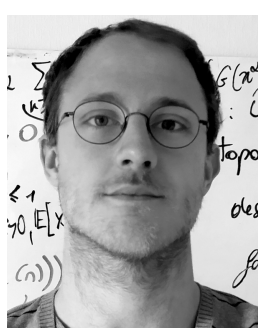

Julien Fageot received the degree from École Normale Supérieure, Paris, France, in 2012, the M.Sc. degree in mathematics from the Université Paris-Sud, France, in 2009, and the M.Sc. degree in imaging science from the École Normale Supérieure, Cachan, France, in 2011. He is currently pursuing the Ph.D. degree with the Biomedical Imaging Group under the direction of M. Unser. He is mainly working on random processes and their applications to signal processing. His research interests include stochastic models for sparse signals and spline

theory.

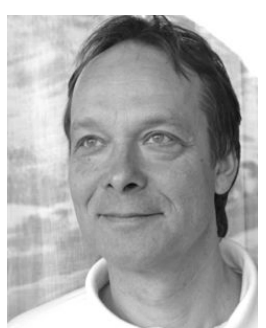

Michael Unser (M'89-SM'94-F'99) is currently a Professor and the Director of Biomedical Imaging Group with the École Polytechnique Fédérale de Lausanne, Lausanne, Switzerland. His primary area of investigation is biomedical image processing. $\mathrm{He}$ is internationally recognized for his research contributions to sampling theory, wavelets, the use of splines for image processing, stochastic processes, and computational bioimaging. He has authored over 250 journal papers on those topics. He has authored the book entitled An Introduction to Sparse Stochastic Processes (Cambridge University Press, 2014) with P. Tafti. From 1985 to 1997, he was with the Biomedical Engineering and Instrumentation Program, National Institutes of Health, Bethesda, USA, conducting research on bioimaging.

He was the Associate Editor-in-Chief (2003-2005) of the IEEE TRANSACTIONS ON MEDICAL IMAGING. He is a member of the Editorial Boards of SIAM Journal on Imaging Sciences, the IEEE Journal on Selected Topics in Signal Processing, and Foundations and Trends in Signal Processing. He is the Founding Chair of the Technical Committee on Bio Imaging and Signal Processing of the IEEE Signal Processing Society.

Prof. Unser was a EURASIP Fellow (2009), and is a member of the Swiss Academy of Engineering Sciences. He is a recipient of several international prizes, including three IEEE-SPS Best Paper Awards and two Technical Achievement Awards from the IEEE (2008 SPS and EMBS 2010). 